\title{
Circular RNA circASPM Promotes the Progression of Glioblastoma by Acting as a Competing Endogenous RNA to Regulate miR-130b-3p/E2F1 Axis
}

\section{Dianqi Hou}

Shanghai general hosiptal

Zhenlin Wang

Shanghai General Hospital

Haimeng Li

Shanghai Zhoupu Hospital

Juan Liu

Shanghai General hospital

Yaohua Liu

Shanghai general hospital

Yang Jiang ( $\square$ windjy0523@tongji.edu.cn )

Shanghai Tenths People's Hospital https://orcid.org/0000-0001-8947-0604

\section{Meiqing Lou}

Shanghai General Hospital

\section{Primary research}

Keywords: Glioblastoma, circASPM, Glioma stem cells, miR-130b-3p, E2F1

Posted Date: December 28th, 2020

DOI: https://doi.org/10.21203/rs.3.rs-132678/v1

License: (a) (i) This work is licensed under a Creative Commons Attribution 4.0 International License. Read Full License

Version of Record: A version of this preprint was published at Journal of Cancer on January 1st, 2022. See the published version at https://doi.org/10.7150/jca.57691. 


\section{Abstract}

background: Glioblastoma Multiform (GBM) is the primary malignancy with the highest incidence and worst prognosis in the adult CNS. Circular RNAs (circRNAs) are a novel and widely diverse class of endogenous non-coding RNAs that can promote or inhibit gliomagenesis. Our study aimed to explore the role of circASPM in GBM and its molecular mechanism.

Methods: Levels of circASPM, miR-130b-3p and E2F1 were determined by quantitative real-time PCR (qRTPCR) or western blotting assay. MTS, Edu, neurospheres formation and extreme limiting dilution assays were used to detect the tumorigenesis and proliferation of GSCs in vitro. The interactions between miR130b-3p and circASPM or E2F1 was demonstrated via qPCR, western blotting, dual-luciferase reporter and RNA immunoprecipitation (RIP) assays. Xenograft experiments was used to analyze tumor growth in vivo.

Results: CircASPM was overexpressed in GBM and could promote the tumorigenesis and proliferation of GSCs both in vitro and in vivo. Mechanistically, circASPM up-regulated the expression of E2F1 in GSCs via miR-130b-3p sponging. We furtherly demonstrated that circAPSM could promote the GSCs proliferation via E2F1 up-regulating. Therefore, our study identified a novel circRNA and its possible mechanism in the development and tumorigenesis of GBM.

Conclusions: CircASPM can promote GBM progression via regulating miR-130b-3p/E2F1 axis, suggesting that circAPSM could provide an effective biomarker for GBM diagnosis and prognostic evaluation and possibly being used for molecular targeted therapy.

\section{Introduction}

Gliomas are the primary malignancies with the highest incidence in the adult CNS, accounting for approximately $70 \%$ of all intracranial primary tumors (1). Especially Glioblastoma Multiform (GBM) has a median survival of fewer than 15 months $(2,3)$. The main current treatment options for GBM include surgical resection combined with chemotherapy (temozolomide, etc.) and local radiotherapy $(4,5)$. Immunotherapy options such as monoclonal antibodies and dendritic cell vaccines are also options, but the results are still unsatisfactory. Glioma stem cells (GSCs) exist in GBM, which have tumor initiation, multi-directional differentiation potential and self-renewal ability, leading to tumor cell infiltrative growth, strong aggressiveness and a high likelihood of radiotherapy resistance, so GBM patients are prone to relapse after treatment and are difficult to be cured, ultimately leading to poor prognosis $(6,7)$. Recent studies have confirmed that the occurrence and development of GBM are closely related to the aberrant expression of various oncogenes or tumor suppressor genes (8), and gene-targeted therapy has been tremendously developed, so identifying the relevant genes in GBM that promote the malignant phenotype of GSCs will help to reveal the biological mechanisms of GBM, refine therapeutic strategies, and improve patient prognosis. 
With the development of sequencing technology, it has been found that coding genes account for less than $10 \%$ of the human genome, while non-coding genes are the majority, and these transcripts are called non-coding RNAs (ncRNAs) (9). Circular RNAs (circRNAs) are a novel and widely diverse class of endogenous ncRNAs, thousands of which exist in mammalian cells, which unlike linear RNAs, form themselves into a covalently closed continuous loop with no $5^{\prime}$ or 3 ' polarity $(10,11)$. CircRNAs have multiple pathways to exert biological effects, such as mediating transcription, interacting with RNAbinding proteins, acting as competitive endogenous RNAs (ceRNAs) (12-14), or play the role of microRNAs (miRNAs) sponges to regulate transcription and modify parental gene expression (11). Dysregulation of circRNAs expression in cancer can promote or inhibit cancer proliferation, tumorigenesis, apoptosis, and angiogenesis (15). Recent studies have confirmed that multiple circRNAs play significant roles in the course of GBM. For example, circ-0014359 promotes GBM progression by targeting the miR-153/PI3K signaling pathway (16), and circHIPK3 regulates the developmental malignant phenotype of GBM by targeting miR-654 from IGF2BP3(17). Through bioinformatics analysis and clinical specimen detection, we found that circASPM (circBase ID: hsa_circ_0015772, chr1:197099044-197113230) was importantly overexpressed in GBM tissues with lower patient survival. However, the cancer-promoting function of circASPM has not yet been reported in the academic community.

MicroRNAs are a highly conserved group of small endogenous non-coding RNAs (19-25 nucleotides in length) in eukaryotes (18), It plays a key role in regulating proliferation, differentiation and apoptosis by binding to the 3'-untranslated regions of downstream mRNAs (19). Existing studies have demonstrated that aberrant miRNAs expression is associated with the initiation and development of various types of tumors $(20,21)$, and it also contributes to the course of GBM (22). Alterations in miRNA expression can positively or negatively regulate tumor progression (23). A series of miRNAs such as miR-15b, miR-21, miR-148a, and miR-196 have also been used as important biomarkers to predict GBM prognosis (24). MiR-130b-3P can act as a tumor suppressor in many tumors, such as nephroblastoma and breast cancer $(25,26)$. MiR-130b-3p is up-regulated in GBM and inhibits cell proliferation, invasion and migration (27).

Our study revealed that circASPM is overexpressed in GBM and can up-regulate E2F1 via miR-130b-3 sponging through bioinformatics analysis. Cellular experiments based on patient-derived GSCs and intracranial tumorigenesis experiments confirmed circASPM could promote GBM initiation, proliferation and the malignant phenotype. Thus, this study identifies circASPM as a novel circRNA associated with the promotion of GBM disease progression and provides a new therapeutic strategy and theoretical basis for the treatment of GBM.

\section{Materials And Methods}

\section{Patient samples and ethics}

We collected 55 clinical samples of glioma patients between January 2010 to December 2014 at the Shanghai General Hospital. 15 samples of grade II, 20 samples of grade III, and 20 samples of grade IV 
glioma (glioblastoma) were included. During the same period, 10 acute brain injury patient samples were collected as a control group. Clinical information for these samples is outlined in Table S1. This study was approved by the Ethics Committee of the Shanghai General Hospital, and written informed consent was obtained from each patient. All animal experiments were conducted under the supervision of the Animal Ethics Committee of Shanghai Jiao Tong University School of Medicine.

\section{Cell treatment and GSCs isolation}

Patient-derived GSCs were isolated and neurosphere cultures were performed as previously reported(6, 28). In brief, fresh clinical glioblastoma specimens were dissociated into single cells and cultured in Dulbecco's modified Eagle's medium (DMEM) with B27 (1:50), recombinant human (rh) basic fibroblast growth factor $(20 \mathrm{ng} / \mathrm{mL})$, and rh-epidermal growth factor $(20 \mathrm{ng} / \mathrm{mL}$, Gibco, Gaithersburg, MD, USA). Neurospheres were then collected and routinely cultured in the above-mentioned neurosphere medium. The cancer stem cell nature of isolated GSCs was confirmed by self-renewal and functional assays of tumor formation in vivo. The expression of stem cell markers (CD133 and nestin+) and the multi-lineage differentiation capacity of GSCs were detected by immunofluorescence. The detailed clinicopathological information is presented in Supplementary Table 2.

\section{Lentiviral vector construction and transfection}

The artificial repeats and whole sequence of circASPM and E2F1 were subcloned into pcDNA3.1 circRNA mini vector and pcDNA3.1 vector. GenePharma (Shanghai, China) engineered siRNA sequences targeted to circASPM and E2F1 to silence them: circASPM-KD1: F: 5'- AAUGGUAUCCCAAAGACUCCU-3', R: 5'GAGUCUUUGGGAUACCAUUAA-3', circASPM-KD2: 5'- UCUUCUUUUUAAUGGUAUCCC-3', R: 5'GAUACCAUUAAAAAGAAGAAA-3'. E2F1-KD: F: 5'- AAAUAACGCUCCAUUAAAGCU-3', R : 5'CUUUAAUGGAGCGUUAUUUAU-3'. The miR-130b-3p mimic, inhibitor, and their negative controls were obtained from Thermo Fisher Scientific (Assay ID: MC23984 and MH23984; Thermo Fisher Scientific, Waltham, MA, USA). All cells were examined for resistance to puromycin (Sigma, Santa Clara, CA, USA) for 15 days at a $10 \mu \mathrm{g} / \mathrm{ml}$ concentration after transfection. Mimics and inhibitor of miR-130b-3p were also purchased from GenePharma.

\section{Quantitative real-time PCR (qRT-PCR)}

TRIzol Reagent (Invitrogen, Carlsbad, CA, USA) was used for RNA extraction. Use M-MLV reverse transcriptase (Invitrogen) or miScript reverse transcription kit (QIAGEN, Hilden, Germany) to synthesize complementary DNA with $1 \mu \mathrm{gRNA}$ as template. The relative expression of circASPM, ASPM mRNA, miR$130 \mathrm{~b}-3 p$ and E2F1 was calculated through $2^{-\Delta \Delta C t}$ method. Glyceraldehyde-3-phosphate dehydrogenase ( $\beta$-actin; for circASPM, ASPM mRNA and E2F1) and U6 (for miR-130b-3p) were used as an internal reference. The sequences of the PCR primer pairs were as follows: circASPM, forward $5^{\prime}$ TCGCCTACTTTGGAATCCTG-3' and reverse 5' - GGCCTTCTTTGAGTGGTGTC-3'; E2F1, forward 5' ACGCTATGAGACCTCACTGAA-3' and reverse 5' - TCCTGGGTCAACCCCTCAAG-3'.

\section{RNase R treatment}


To determine the stability of circASPM, $10 \mu \mathrm{g}$ RNA extracted from the patient-derived cell lines was incubated with RNase R (4U/ $\mu$ g; Epicentre Biotechnologies, Madison, WI, USA) or not for 1 hour at $37^{\circ} \mathrm{C}$. Then qRT-PCR was used to detect the relative expression of circASPM and ASPM mRNA.

\section{Western blotting}

Western blotting was performed as described previously(29). The protein samples were prepared by a total cell protein extraction kit (Vazyme, Nanjing, China). BCA protein quantification kit (Vazyme) was used for concentration determination. $40 \mu \mathrm{g}$ protein samples were transferred to polyvinylidene fluoride membranes (Thermo Fisher Scientific) after sodium dodecyl sulfate-polyacrylamide gel electrophoresis and then blocked with fat-free milk. The primary antibodies against E2F1 (1:1000, \#ab112580, Abcam, Cambridge, UK), $\beta$-actin (1:2000, \#66009-1-Ig, ProteinTech, Chicago, IL, USA) were used. ECL kits (Beyotime Biotechnology, Beijing, China) and IMAGE J software (National Institutes of Health, Bethesda, $M D, U S A)$ were used to quantify the signal intensity.

\section{Immunofluorescence}

Immunofluorescence was performed as described previously (30). Cells were fixed, permeabilized and blocked with $4 \%$ paraformaldehyde, $0.5 \%$ Triton X-100, and $5 \%$ BSA, incubated with primary antibody overnight at $4{ }^{\circ} \mathrm{C}$, followed with FITC- or rhodamine-conjugated secondary antibody. The antibodies against CD133 (1:1000, \#ab222782, Abcam), nestin+ (1:500, \#ab18102, Abcam), GFAP (1:1000, \#ab4674, Abcam) and $\beta$ III tubulin (1:1000, \#ab7751, Abcam) were used. DAPI (Sigma, Santa Clara, CA, USA) is acquired to stain cell nuclei. A laser scanning confocal microscope (IX71, Olympus, Tokyo, Japan) was used to photograph the expression of immunofluorescence.

\section{MTS proliferation assay}

According to the manufacturer's protocol, the CellTiter 96 Aqueous non-radioactive cell proliferation detection kit (Promega, Madison, WI, USA) was used to evaluate GCS proliferation. An ultraviolet spectrophotometer (Thermo Fisher Scientific) was used to measure the absorption at $495 \mathrm{~nm}$.

\section{5-Ethynyl-2'-deoxyuridine (EdU) proliferation assay}

EdU assays were performed as previously described(31). In brief, cell lines were seeded in 24-well plates at a density of $1 \times 10^{5}$ cells per well for $24 \mathrm{~h}$ before being added with EdU reagent (Beyotime Biotechnology, Beijing, China) for 2 hours at $37^{\circ} \mathrm{C}$. The cells were fixed with paraformaldehyde, and the percentage of EdU positive cells was calculated by a laser scanning confocal microscope (Olympus).

\section{Limiting Dilution Neurosphere Formation Assay}

The self-renewal ability of GSCs was assessed by the neurosphere formation assay as reported previously (28). Briefly, GSCs were dissociated and seeded into 24-well plates and incubated in fresh medium for 7 days. The relative size and number were counted by an optical microscope (Olympus). In vitro limiting dilution method, GSCs were seeded at a gradient of 1,10,20,30, 40 or 50 cells per well into 
96-well plates for 7 days. Later, the number of neurospheres in each well was analyzed and calculated as previously described (32).

\section{Luciferase activity analysis}

Luciferase reporter assays were performed as previously described (33). Briefly, wild type circASPM, mutant circASPM, wild-type E2F1 and mutant E2F1 were cloned into the empty pmiRGLO luciferase reporter vector (Promega). Then the Dual-Luciferase Reporter Assay System (Promega) was used to detect luciferase activity according to the manufacturer's protocol. Each experiment was independently repeated three times.

\section{RNA immunoprecipitation (RIP) assay}

RIP assay was performed with the EZ-Magna RIP RNA-binding Protein Immunoprecipitation Kit (Millipore, Darmstadt, Germany). GSCs in different conditions were lysed with RIP lysis buffer and magnetic beads conjunct with antibodies against Ago2 and antibodies against IgG, which was used as a negative control. The immunoprecipitated RNAs were isolated after incubation with proteinase K. Finally, The expressions of circASPM, miR-130b-3p and E2F1 were calculated through qRT-PCR.

\section{Xenograft experiments}

Transfected GSCs were injected ( $5 \times 10^{4}$ cells per mice) orthotopically into the brains of 6-week-old female BALB/c nude mice (Beijing Vital River Laboratory Animal Technology, Beijing, China). The injection point was $2 \mathrm{~mm}$ lateral and $2 \mathrm{~mm}$ anterior to the bregma, determined with a stereotaxic apparatus. We observed mice daily for neurological symptoms or death, and the tumor volume was measured according to the formula: $V=\left(D \times d^{2}\right) / 2$, that $D$ was the longest diameter and $d$ was the shortest diameter of the tumor. When neurological symptoms were observed, mice were sacrificed by cervical spine dislocation, and the brains were collected for analysis as previously reported (33). All animal experiments were performed according to the guidelines of the Animal Care Committee of Shanghai Jiao Tong University School of Medicine.

\section{Immunohistochemistry (IHC)}

IHC of mice xenograft tumor specimens was performed as previously described (30). In short, tissue samples embedded in paraffin were cut into $4 \mu \mathrm{m}$ sections and labeled with a primary antibody against E2F1, ki-67. The sections were imaged using an optical microscope (Olympus), and the staining intensity was evaluated according to the German immunohistochemical scoring system (34).

\section{Bioinformatic analyses}

The data of circRNA expression in gliomas was obtained from Gene Expression Omnibus (GEO) datasets GSE109569. Starbase (starbase.sysu.edu.cn) was used to predict the binding sites between miR-130b-3p and circASPM or E2F1.

\section{Statistical analysis}


SPSS 22.0 software (IBM, Armonk, NY, USA) was used for the statistical analysis of this study. All experiments were repeated more than 3 times, and the results were expressed as mean \pm standard error. The statistical significance between groups was tested by the chi-square test and t-test. The Log-rank test and Kaplan-Meier analysis were used to analyze differences in survival rates. All statistical tests were two-sided tests, and statistical significance is defined as $P$ value $<0.05$.

\section{Results}

\section{CircASPM is up-regulated in glioblastomas tissues and correlated with the progression and poor prognosis.}

CircASPM is derived from transcript 1 of the ASPM gene (chr1:197099044-197113230) and is formed into a loop by reverse splicing its exons 1-7 (Fig. 1b). We first found that the expression of circASPM was significantly higher in glioma tissues than in normal tissues based on Gene Expression Omnibus (GEO) datasets GSE109569 (Fig. 1a, d). To validate the bioinformatics analysis's conclusions, we selected clinical specimens from 55 glioma patients for QPCR assays and confirmed that circASPM expression was up-regulated with increasing WHO grade (Fig. 1C). Subsequently, we performed a Kaplan-Meier survival analysis on the prognostic significance of circASPM expression in 55 patients. The results showed that the average survival time of patients with high circASPM expression was significantly shorter than those with low expression (qPCR quantification, Cutoff: median, Fig. 1g). The clinical information of the above 55 samples is outlined in Supplementary Table 1.

We further extracted patient-derived GSCs from fresh clinical GBM specimens for culture, from which we selected six GSCs in the best growth state for subsequent studies. Supplementary Table 2 shows that the clinical information about the patients from whom the six GSCs were derived. In Supplementary Fig. 1a, we show hematoxylin and eosin (H\&E) staining of the original patient tumors. Immunofluorescence staining was performed to confirm the expression of the stem cell markers CD133 + and Nestin + in the isolated neurospheres (Sup. Figure 1b). Moreover, Supplementary Fig. 1c fully demonstrates the multilineage differentiation capacity of GSCs. We then verified the stability of circASPM using RNase $R$ assays. RNase R assays are commonly used to confirm RNAs' circular structure because it can degrade linear RNAs with short 3'-tails, whereas it cannot degrade circRNAs (35). After RNase R disposition, ASPM mRNA expression was significantly decreased in GSC11 and GSC18 cells, while circASPM was unaffected (Fig. 1e, f), indicating that circASPM is more resistant to RNase R digestion. Subsequently, we applied qPCR to determine the expression of circASPM in six cell lines, with GSC11 having the lowest expression and GSC18 the highest (Sup. Figure 1d). In summary, circASPM is a stable circRNA that is potentially involved in promoting glioblastomas' malignant phenotype.

\section{Overexpression of circASPM promoted the proliferation of GSCs in vitro.}

We performed a further series of gain-of-function assays to demonstrate the role of circASPM in GSCs, and we designed lentiviral-coated circASPM overexpression plasmids to infect GSC11 and GSC12, respectively, based on their low expression levels of circASPM (Sup. Figure 1d). qPCR confirmed that 
circASPM overexpression was optimal (Fig. 2a). First, we found by MTS assays that the absorbance values of GSCs after circASPM overexpression were significantly higher than those of controls (Fig. 2b, c), confirming that circASPM overexpression promoted the viability of GSCs. Next, we used Edu assays to examine the role of circASPM overexpression on tumor proliferative capacity. The proportion of Edupositive cells in circASPM overexpressing cell lines was higher than that in controls, indicating that tumor cells' proliferative capacity was promoted after circASPM overexpression (Fig. 2d-f). Furthermore, in our neurosphere formation experiments, the relative size of spheres and the number of spheres formed per unit time after circASPM overexpression were greater than those in the EV group (Fig. 2g, h). Extreme limiting dilution assay also showed that overexpression of circASPM increased the rate of tumor formation (Fig. 2i, j). The above results showed that circASPM overexpression could promote the proliferation of glioma stem cells.

\section{Knockdown of circASPM suppressed the proliferation of GSCs in vitro.}

To continue investigating the function of circASPM in the malignant phenotype of GSCs, we knocked down circASPM by transfecting si-circASPM into GSCs and used QPCR to confirm the efficiency of circASPM knockdown (Fig. 3a). We first used MTS assays to find a significant decrease in absorbance values after circASPM knockdown, demonstrating that tumor viability was significantly inhibited (Fig. 3b, c). Subsequently, in Edu assays, we found that knockdown of circASPM significantly down-regulated the proportion of Edu-positive cells (Fig. 3d-f). Besides, the proliferative activity of neurospheres after circASPM knockdown was found to be significantly inhibited in neurosphere formation assays (Fig. $3 \mathrm{~g}, \mathrm{j}$ ). The extreme limiting dilution assays also showed a significant decrease in tumor formation after knockdown of circASPM compared to the NC group (Fig. 2h, i). The above results indicate that knockdown of circASPM can suppress the malignant phenotype of GSCs and induce their apoptosis.

\section{MiR-130b-3p could bind with circAPSM and mediated the function of GBM cells.}

Existing studies suggest circRNAs have many microRNA response elements (MREs), and usually exert biological functions through miRNA sponging and regulate the target genes (2). To investigate the interaction between miRNAs and circAPSM, we searched the Starbase dataset and found a binding site for miR-130b-3p to circAPSM (Fig. 4a). We subsequently confirmed by qPCR assays that the expression of circASPM was up-regulated in GSC11 and GSC12 with the administration of miR-130b-3p inhibitor, whereas the opposite result was obtained in GSC17 and GSC18 with the administration of miR-130b-3p mimic (Fig. 4b, c). Further, we examined the relative expression of miR-130b-3p by qPCR assays after overexpression and knockdown of circASPM. MiR-130b-3p expression was also negatively regulated by circASPM levels (Fig. 4d, i). We then utilized luciferase reporter assays and found increased luciferase activity of wild-type circASPM in GSC11 and GSC12 after miR-130b-3p inhibitor treatment (Fig. 4e, f). In contrast, miR-130b-3p mimic treatment reduced the luciferase activity of wild-type circASPM luciferase in GSC17 and GSC18 (Fig. 4g, h). Since miRNAs bind to MREs via the RNA-induced silencing complex (RISC), and the Ago 2 (AGO2) protein is a key component of RISC (36), we performed anti-AGO2 RIP assays to determine whether miR-130b-3p and circAPSM are co-enriched in RISC, in which anti-AGO2 
antibodies effectively pulled down both circASPM and miR-130b-3p compared to IgG. In addition, both circASPM and miR-130b-3p also showed significant enrichment after miR-130b-3p mimic treatment compared to miR-130b-3p negative control (Fig. 4m, p). In summary, miR-130b-3p is likely to bind with circAPSM, and the changes in their expression can negatively regulate each other's expression.

We then performed a series of experiments to verify that miR-130b-3p could mediate GBM cells' function after binding with circAPSM. MTS assays revealed that circAPSM knockdown in GSC18 could inhibit proliferation. However, miR-130b-3p inhibitor treatment resulted in stronger tumor proliferation. In contrast, the pro-tumorigenic effect produced by overexpression of circAPSM completely disappeared after miR-130b-3p mimic treatment in GSC11, and inhibition even occurred compared to the control (Fig. 4j, k). In Edu assays, the proportion of Edu-positive cells that decreased due to knockdown of circASPM increased substantially after miR-130b-3p inhibitor treatment. In contrast, the increase in the proportion of Edu-positive cells due to circASPM overexpression decreased significantly after mimic treatment of miR-130b-3p (Fig. 4m, n, p). Neurosphere formation assays similarly revealed that knockdown treatment of circASPM resulted in GSC18-generated neurospheres with relatiely smaller size and fewer numbers. That neurosphere formation was excessively promoted after treatment with miR130b-3p inhibitor. In contrast, circASPM overexpression induced GSC11 to produce more and larger neurospheres. After treatment with miR-130b-3p mimic, the size and number of neurospheres were reduced to be even smaller than the control (Fig. 4q, t). Extreme limiting dilution assays also showed a similar trend (Fig. $4 r, s)$. These data suggest that miR-130b-3p suppresses the malignant phenotype of GBM by inhibiting circAPSM expression in GSCs.

\section{CircAPSM regulated E2F1 expression via miR-130b-3p sponging.}

Next, we intend to search for functional targets of miR-130b-3p to determine the mechanism by which circAPSM sponging with miR-130b-3p exerts biological function. We determined by StarBase 3.0 that the 3'-UTR of E2F1 has a binding site with miR-130b-3p (Fig. 5a). Luciferase reporter experiments showed that inhibitor treatment of miR-130b-3p triggered an approximate doubling of the luciferase activity of E2F1-wt compared to the E2F1-mt group. In contrast, the luciferase activity of E2F1-wt was significantly decreased after miR-130b-3p mimic treatment (Fig. 5b-e). Both qPCR assays and western blotting showed a significant increase in E2F1 expression after miR-130b-3p inhibitor treatment in GSC11 and GSC12, and a decrease in E2F1 expression after miR-130b-3p mimic treatment in GSC17 and GSC18 (Fig. 5f-k). Finally, we again performed qPCR and western blotting to explore the effect of circASPM on E2F1 expression via miR-130b-3p sponging, and the results demonstrated that knockdown and overexpression treatment of circASPM in GSC18 and GSC11 could positively regulate the expression of E2F1. Meanwhile, the expression level of E2F1 was significantly reversed after rescue experiments with miR-130b-3p inhibitor and mimic treatment, respectively (Fig. 5l,m,n). The above results prove that circAPSM regulates E2F1 expression through miR-130b-3p sponging.

\section{CircAPSM mediated GSCs proliferation via E2F1 expression.}


Since circASPM acts as a sponge of miR-130b-3p to regulate E2F1 expression, we further designed a series of experiments to determine whether circASPM up-regulates E2F1 expression in GSCs via the miR130b-3p-mediated ceRNA mechanism to promote the malignant phenotype of tumors. MTS assays (Fig. 6a, b), EDU assays (Fig. 6c-e), neurosphere formation assays (Fig. 6f, i) and extreme limiting dilution assays (Fig. $6 \mathrm{~g}, \mathrm{~h}$ ) demonstrated that knockdown treatment of circASPM in GSC18 significantly decreased the viability, proliferation capacity and formation rate of neurospheres. On the contrary, circASPM overexpression treatment of GSC11 up-regulated tumor cells' viability, proliferation capacity, and neurospheres formation rate. Then, we further performed additional E2F1 knockdown or overexpression treatment on the base of circAPSM overexpression or knockdown, respectively. The results showed an over-reversal compared to the levels without the circASPM knockdown or overexpression treatment after manipulating the levels of E2F1. Taken together, these results strongly suggest that circASPM plays a biological role in regulating E2F1 expression through the miR-130b-3pmediated ceRNA mechanism and promotes the malignant phenotype of GBM by up-regulating E2F1 expression in GSCs.

\section{CircAPSM promoted GSCs tumorigenesis in vivo.}

We explored the role of circAPSM in vivo by establishing a tumor xenograft model. We found that knockdown of circAPSM resulted in a significant reduction in GSC18 tumor size (Fig. 7a, C) and a significantly longer median survival time (mean survival: $20.6 \pm 6.19$ and $29 \pm 7.18$ days; Fig. 7c). Comparatively, overexpression of circAPSM significantly promoted intracranial tumor growth in GSC11 (Fig. 7d, e) and reduced survival time (mean survival: $25.4 \pm 6.50$ days, $16.2 \pm 6.02$ days; Fig. $7 f$ ) compared to controls. By further H\&E staining and immunohistochemical staining, we found that the staining intensities and expression levels of circAPSM, E2F1 and ki-67 were significantly higher in the circAPSM overexpression group than in the control group, while we obtained opposite results in the knockdown group. The above results demonstrated that circAPSM promoted GSCs tumorigenesis in vivo.

\section{Discussion}

The World Health Organization (WHO) classifies gliomas into four grades according to tumor cell morphology, including WHO grade I, II, III, IV. The most malignant grade IV glioma is also known as GBM $(37,38)$, and more than half of all gliomas are GBM $(1)$. The current traditional GBM treatment plan of surgical resection combined with radiotherapy and chemotherapy has a poor prognosis, such as a high postoperative recurrence rate and short survival time $(39,40)$. Therefore, an in-depth study of the course of GBM is imperative. Many recent studies have found that circRNAs can exert their biological effects through multiple pathways and pay contributions to the course of GBM (12-14). This study aims to validate the tumorigenic role of circASPM in GBM and detect the mechanisms by which it exerts its functions.

GEO analysis revealed that circASPM was expressed at higher levels in GBM compared to normal tissue and low-grade gliomas and that its expression level was positively correlated with patient survival time. 
We then cultured patient-derived GSCs cell line and used RNase R assays to prove that circASPM was stable after verifying that the cell lines culture worked well (35). Subsequently, we performed gain-offunction assays and loss-of-function assays to analyze the biological role of circASPM in GBM progression. We found that the expression level of circASPM can positively regulate the proliferation and growth of tumor cells, suggesting that circASPM plays an important regulatory role in the development of GBM.

Many researchers have found very complex links and interactions between different types of RNA (10). CircRNA can act as a ceRNA to regulate target genes' expression through miRNA sponging $(11,41)$. We found a binding site for miR-130b-3p on circAPSM by searching StarBase 3.0 in our study, and subsequent assays also proved our prediction. According to our data, the expression of miR-130b-3p was inversely correlated with circASPM in vivo. In addition, miR-130b-3p inhibitor treatment and mimic treatment could eliminate the promoting or inhibiting effects of circASPM overexpression or knockdown, respectively.

It has long been reported that miRNAs can bind to the 3'-UTR of mRNA to perform their own biological functions (42). So we further probed and found that E2F1 is a direct target of miR-130b-3p. E2F1 mediates regulation of the cell cycle, and its aberrant activation is strongly associated with poor prognosis in a variety of cancers $(43,44)$. E2F1 pays great contributions to promote the development and maintains the stemness of tumor cells in gastric cancer, prostate cancer or some other tumors $(45,46)$. In glioma, E2F1 can be used as a target for miR-205, miR-371 to promote glioma growth and invasion (47, 48). Our results showed that both mRNA and protein levels of E2F1 were up-regulated in GSCs tissues and cells in response to circASPM overexpression, exerting stronger tumor-promoting effects. Subsequent rescue experiments of E2F1 reversed the biological effects produced by circASPM overexpression or knockdown, corroborating that circASPM regulates E2F1 expression by acting as a ceRNA for miR-130b$3 p$.

Finally, we performed xenograft experiments and found that overexpression of circASPM significantly promoted the growth of intracranial tumors and shortened the survival time of mice. The staining intensity and expression levels of circAPSM, E2F1, and ki-67 were significantly higher than those in the control group, whereas in the knockdown group, we obtained the opposite results. These data mentioned above prove that circAPSM promotes GSCs tumorigenesis in vivo. However, this study lacks depth in exploring more specific mechanisms such as angiogenesis, signaling pathways, etc., and warrants continued follow-up studies.

\section{Conclusion}

In conclusion, this study demonstrates that circASPM is highly expressed in GBM, which up-regulates the expression level of E2F1 by acting as a ceRNA for miR-130b-3p, promotes a range of malignant behaviors such as growth, proliferation and invasion, and leads to a poor patient prognosis. Subsequent studies and clinical translation against circASPM would be valuable. 


\section{Abbreviations}

CircRNAs: Circular RNAs; ncRNAs: non-coding RNAs; ceRNAs: endogenous RNAs; miRNAs: microRNAs; WHO: World Health Organization; GBM: Glioblastoma multiforme; GSCs: Glioma stem cells; MREs: microRNA response elements; GEO: Gene Expression Omnibus; H\&E stain: hematoxylin and eosin stain; IHC: Immunohistochemistry; qRT-PCR/qPCR: Real-Time Quantitative Reverse Transcription PCR; RISC: RNA-induced silencing complex.

\section{Declarations}

\section{Ethics approval and consent to participate}

The present study was approved by the ethical review committee of Shanghai General Hospital of Shanghai Jiao Tong University School. Written informed consent was obtained from all enrolled patients

\section{Consent for publication}

Patients agree to participate in this work.

\section{Availability of data and materials}

The analyzed data sets generated during the present study are available from the corresponding author on reasonable request.

\section{Competing interest}

The authors declare no conflicts of interest.

\section{Funding}

This work was supported by the National Natural Science Foundation of China (81801214).

\section{Authors' contribution}

Yang $\mathrm{J}$ and Meiqing $\mathrm{L}$ participated in the design of the study. Haimeng $\mathrm{L}$ performed the bioinformatics analysis and analyzed the data. Yaohua $L$ and Juan $L$ contributed to data collection and the interpretation of data. Dianqi and Zhenlin W performed the experiments, collected the data and wrote the manuscript. All authors read and approved the manuscript.

\section{Acknowledgements}

Not applicable

\section{References}


1. Ostrom QT, Cioffi G, Gittleman H, Patil N, Waite K, Kruchko C, et al. CBTRUS Statistical Report: Primary Brain and Other Central Nervous System Tumors Diagnosed in the United States in 20122016. Neuro Oncol. 2019;21(Suppl 5):v1-100.

2. Louis DN, Perry A, Reifenberger G, von Deimling A, Figarella-Branger D, Cavenee WK, et al. The 2016 World Health Organization Classification of Tumors of the Central Nervous System: a summary. Acta Neuropathol. 2016;131(6):803-20.

3. Omuro A, DeAngelis LM. Glioblastoma and other malignant gliomas: a clinical review. Jama. 2013;310(17):1842-50.

4. Zhou J, Jiang Y, Zhao J, Zhang H, Fu J, Luo P, et al. Dp44mT, an iron chelator, suppresses growth and induces apoptosis via RORA-mediated NDRG2-IL6/JAK2/STAT3 signaling in glioma. Cell Oncol (Dordr). 2020;43(3):461-75.

5. Lapointe S, Perry A, Butowski NA. Primary brain tumours in adults. Lancet. 2018;392(10145):43246.

6. Su R, Cao S, Ma J, Liu Y, Liu X, Zheng J, et al. Knockdown of SOX2OT inhibits the malignant biological behaviors of glioblastoma stem cells via up-regulating the expression of miR-194-5p and miR-122. Mol Cancer. 2017;16(1):171.

7. Zhao J, Liu P, Ma J, Li D, Yang H, Chen W, et al. Enhancement of Radiosensitization by Silver Nanoparticles Functionalized with Polyethylene Glycol and Aptamer As1411 for Glioma Irradiation Therapy. Int J Nanomedicine. 2019;14:9483-96.

8. Wang Y, Jiang T. Understanding high grade glioma: molecular mechanism, therapy and comprehensive management. Cancer Lett. 2013;331(2):139-46.

9. Morris KV, Mattick JS. The rise of regulatory RNA. Nat Rev Genet. 2014;15(6):423-37.

10. Hou LD, Zhang J. Circular RNAs: An emerging type of RNA in cancer. Int J Immunopathol Pharmacol. 2017;30(1):1-6.

11. Qu S, Yang X, Li X, Wang J, Gao Y, Shang R, et al. Circular RNA: A new star of non-coding RNAs. Cancer Lett. 2015;365(2):141-8.

12. Salmena L, Poliseno L, Tay Y, Kats L, Pandolfi PP. A ceRNA hypothesis: the Rosetta Stone of a hidden RNA. language? Cell. 2011;146(3):353-8.

13. Zeng Y, Du WW, Wu Y, Yang Z, Awan FM, Li X, et al. A Circular RNA Binds To and Activates AKT Phosphorylation and Nuclear Localization Reducing Apoptosis and Enhancing Cardiac Repair. Theranostics. 2017;7(16):3842-55.

14. Yang Y, Gao X, Zhang M, Yan S, Sun C, Xiao F, et al. Novel Role of FBXW7 Circular RNA in Repressing Glioma Tumorigenesis. J Natl Cancer Inst. 2018;110(3):304-15.

15. Yu J, Xu QG, Wang ZG, Yang Y, Zhang L, Ma JZ, et al. Circular RNA cSMARCA5 inhibits growth and metastasis in hepatocellular carcinoma. J Hepatol. 2018;68(6):1214-27.

16. Shi F, Shi Z, Zhao Y, Tian J. CircRNA hsa-circ-0014359 promotes glioma progression by regulating miR-153/PI3K signaling. Biochem Biophys Res Commun. 2019;510(4):614-20. 
17. Jin $P$, Huang $Y$, Zhu P, Zou Y, Shao T, Wang O. CircRNA circHIPK3 serves as a prognostic marker to promote glioma progression by regulating miR-654/IGF2BP3 signaling. Biochem Biophys Res Commun. 2018;503(3):1570-4.

18. Li X, Liu F, Lin B, Luo H, Liu M, Wu J, et al. miR-150 inhibits proliferation and tumorigenicity via retarding G1/S phase transition in nasopharyngeal carcinoma. Int J Oncol. 2017;50(4):1097-108.

19. Khew-Goodall Y, Goodall GJ. Myc-modulated miR-9 makes more metastases. Nat Cell Biol. 2010;12(3):209-11.

20. Hayes J, Peruzzi PP, Lawler S. MicroRNAs in cancer: biomarkers, functions and therapy. Trends Mol Med. 2014;20(8):460-9.

21. Lee YS, Dutta A. MicroRNAs in cancer. Annu Rev Pathol. 2009;4:199-227.

22. Calin GA, Croce CM. MicroRNA signatures in human cancers. Nat Rev Cancer. 2006;6(11):857-66.

23. Lu GF, Geng F, Xiao Z, Chen YS, Han Y, You CY, et al. MicroRNA-6807-3p promotes the tumorigenesis of glioma by targeting downstream DACH1. Brain Res. 2019;1708:47-57.

24. Zhang Y, Chen J, Xue Q, Wang J, Zhao L, Han K, et al. Prognostic Significance of MicroRNAs in Glioma: A Systematic Review and Meta-Analysis. Biomed Res Int. 2019;2019:4015969.

25. Hu Y, Yan J. Aberrant expression and mechanism of miR-130b-3p/phosphatase and tensin homolog in nephroblastoma in children. Exp Ther Med. 2019;18(2):1021-8.

26. Shui Y, Yu X, Duan R, Bao Q, Wu J, Yuan H, et al. miR-130b-3p inhibits cell invasion and migration by targeting the Notch ligand Delta-like 1 in breast carcinoma. Gene. 2017;609:80-7.

27. Xie Y, Cheng Y. Long non-coding RNA CASC15 is up-regulated in glioma and facilitates cell proliferation and metastasis via targeting miR-130b-3p. Eur Rev Med Pharmacol Sci. 2019;23(17):7475-81.

28. Jiang Y, Song Y, Wang R, Hu T, Zhang D, Wang Z, et al. NFAT1-Mediated Regulation of NDEL1 Promotes Growth and Invasion of Glioma Stem-like Cells. Cancer Res. 2019;79(10):2593-603.

29. Guan Y, Cao Z, Du J, Liu T, Wang T. Circular. RNA circPITX1 knockdown inhibits glycolysis to enhance radiosensitivity of glioma cells by miR-329-3p/NEK2 axis. Cancer Cell Int. 2020;20:80.

30. Jiang Y, Han S, Cheng W, Wang Z, Wu A. NFAT1-regulated IL6 signalling contributes to aggressive phenotypes of glioma. Cell Commun Signal. 2017;15(1):54.

31. Jiang Y, Zhou J, Luo P, Gao H, Ma Y, Chen YS, et al. Prosaposin promotes the proliferation and tumorigenesis of glioma through toll-like receptor 4 (TLR4)-mediated NF-KB signaling pathway. EBioMedicine. 2018;37:78-90.

32. Hu Y, Smyth GK. ELDA: extreme limiting dilution analysis for comparing depleted and enriched populations in stem cell and other assays. J Immunol Methods. 2009;347(1-2):70-8.

33. Song $Y$, Jiang $Y$, Tao D, Wang $Z$, Wang $R$, Wang $M$, et al. NFAT2-HDAC1 signaling contributes to the malignant phenotype of glioblastoma. Neuro Oncol. 2020;22(1):46-57.

34. Han S, Zhang C, Li Q, Dong J, Liu Y, Huang Y, et al. Tumour-infiltrating CD4(+) and CD8(+) lymphocytes as predictors of clinical outcome in glioma. Br J Cancer. 2014;110(10):2560-8. 
35. Jiang Y, Zhou J, Zhao J, Zhang H, Li L, Li H, et al. The U2AF2 / circRNA ARF1/miR-342-3p/ISL2 feedback loop regulates angiogenesis in glioma stem cells. J Exp Clin Cancer Res. 2020;39(1):182.

36. Huang W, Fang K, Chen TQ, Zeng ZC, Sun YM, Han C, et al. circRNA circAF4 functions as an oncogene to regulate MLL-AF4 fusion protein expression and inhibit MLL leukemia progression. $\mathrm{J}$ Hematol Oncol. 2019;12(1):103.

37. Louis DN, Ohgaki H, Wiestler OD, Cavenee WK, Burger PC, Jouvet A, et al. The 2007 WHO classification of tumours of the central nervous system. Acta Neuropathol. 2007;114(2):97-109.

38. Wang Z, Ying C, Zhang A, Xu H, Jiang Y, Lou M. HCK promotes glioblastoma progression by TGF $\beta$ signaling. Biosci Rep. 2020;40(6).

39. Van Meir EG, Hadjipanayis CG, Norden AD, Shu HK, Wen PY, Olson JJ. Exciting new advances in neuro-oncology: the avenue to a cure for malignant glioma. CA Cancer J Clin.60(3):166-93.

40. Ostrom QT, Bauchet L, Davis FG, Deltour I, Fisher JL, Langer CE, et al. The epidemiology of glioma in adults: a "state of the science" review. Neuro Oncol. 2014;16(7):896-913.

41. Zhu Z, Yu Z, Rong Z, Luo Z, Zhang J, Qiu Z, et al. The novel GINS4 axis promotes gastric cancer growth and progression by activating Rac1 and CDC42. Theranostics. 2019;9(26):8294-311.

42. Kim D, Sung YM, Park J, Kim S, Kim J, Park J, et al. General rules for functional microRNA targeting. Nat Genet. 2016;48(12):1517-26.

43. Chun JN, Cho M, Park S, So I, Jeon JH. The conflicting role of E2F1 in prostate cancer: A matter of cell context or interpretational flexibility? Biochim Biophys Acta Rev Cancer. 2020;1873(1):188336.

44. Poppy Roworth A, Ghari F, La Thangue NB. To live or let die - complexity within the E2F1 pathway. Mol Cell Oncol. 2015;2(1):e970480.

45. Liang YX, Lu JM, Mo RJ, He HC, Xie J, Jiang FN, et al. E2F1 promotes tumor cell invasion and migration through regulating CD147 in prostate cancer. Int J Oncol. 2016;48(4):1650-8.

46. Enjoji S, Yabe R, Tsuji S, Yoshimura K, Kawasaki H, Sakurai M, et al. Stemness Is Enhanced in Gastric Cancer by a SET/PP2A/E2F1 Axis. Mol Cancer Res. 2018;16(3):554-63.

47. Li FF, Xing C, Wu LL, Xue F. MiR-205 enhances cisplatin sensitivity of glioma cells by targeting E2F1. Eur Rev Med Pharmacol Sci. 2018;22(2):299-306.

48. Xia L, Nie D, Wang G, Sun C, Chen G. FER1L4/miR-372/E2F1 works as a ceRNA system to regulate the proliferation and cell cycle of glioma cells. J Cell Mol Med. 2019;23(5):3224-33.

\section{Figures}



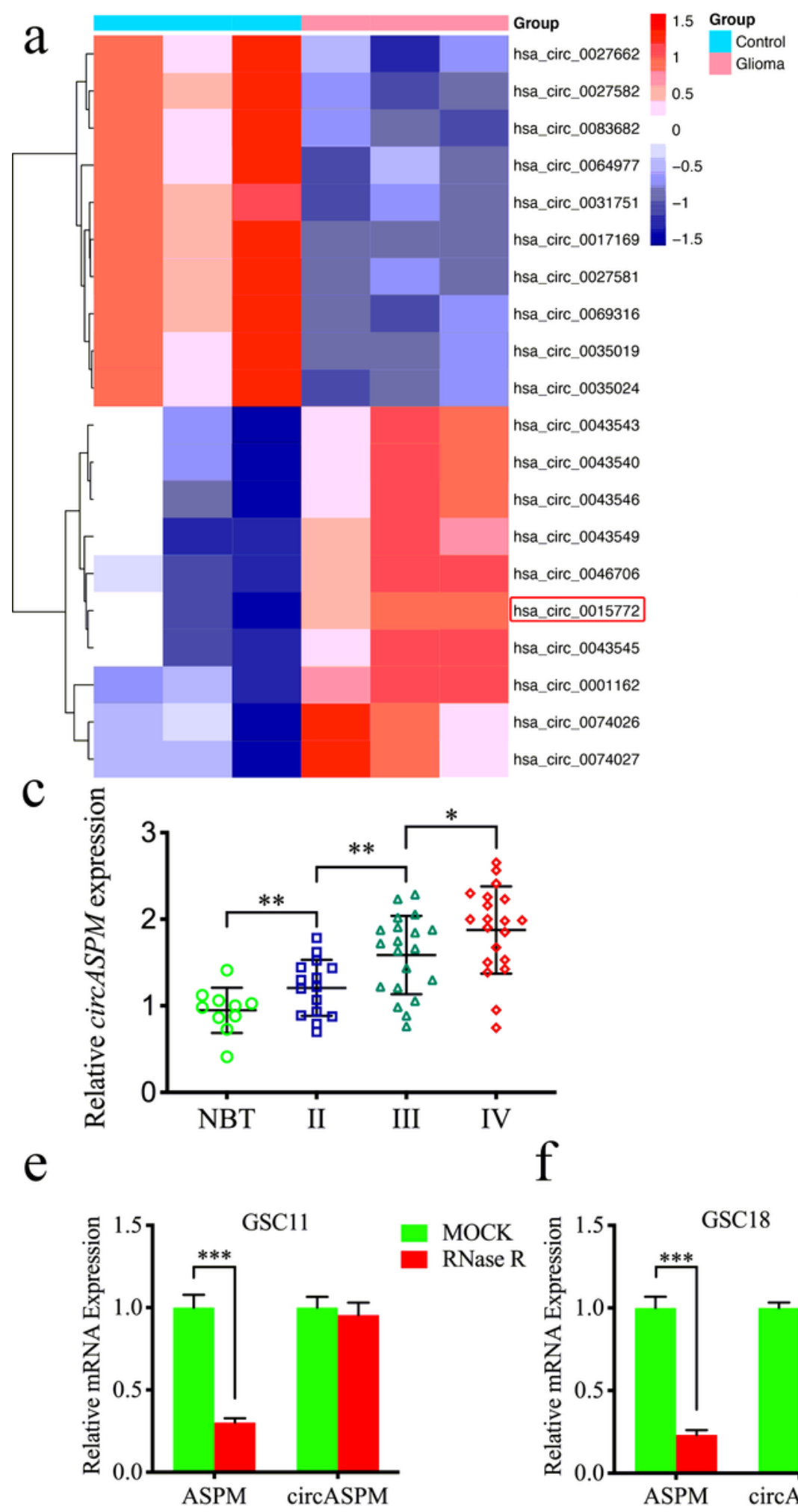

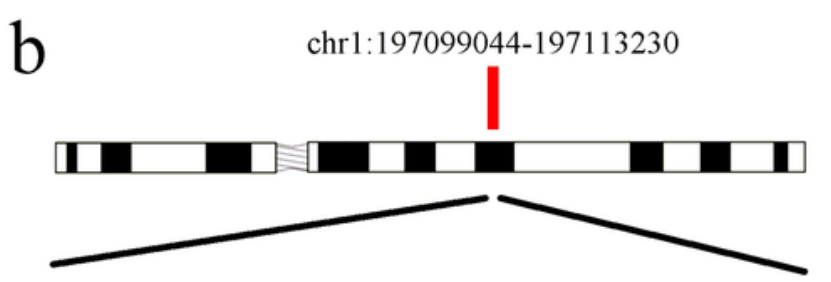

ASPM, transcript variant 1: chr1:197084127-197146694

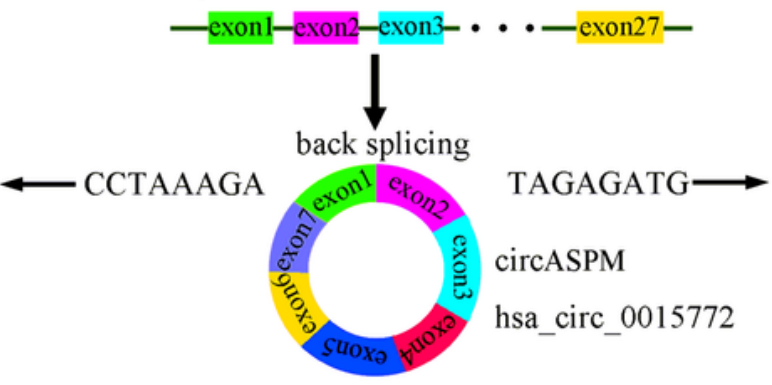

d
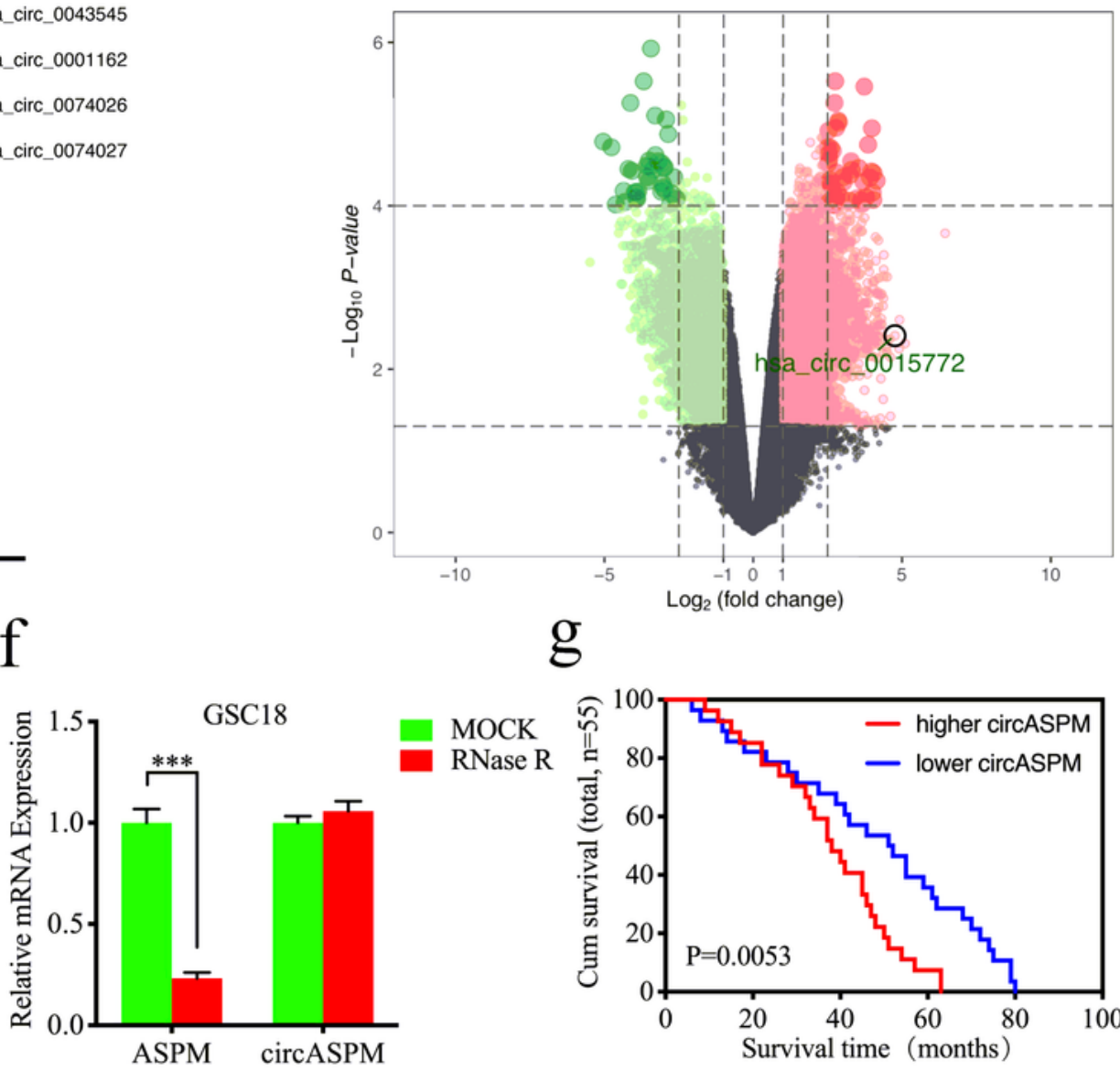

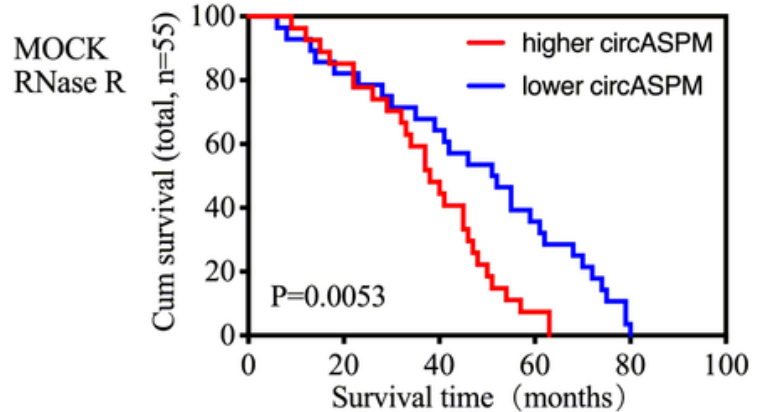

Figure 1

CircASPM is up-regulated in glioblastomas tissues and correlated with the progression and poor prognosis. a, d: CircASPM expression was significantly higher in glioma tissues than in normal brain tissues based on GEO datasets GSE109569. b: Loop formation procedure of circASPM was illustrated. c: CircASPM expression was correlated with WHO grades in GBM e, f: qPCR analysis of ASPM mRNA and circASPM in GSC11 and GSC18 treated with Rnase R. g: Kaplan-Meier analysis of GBM patients in terms 
of higher circASPM expression versus low circASPM expression. All data are shown as the mean \pm SD (three independent experiments). ${ }^{*} \mathrm{P}<0.05 ; * \star \mathrm{P}<0.01$; $* * * \mathrm{P}<0.001$.

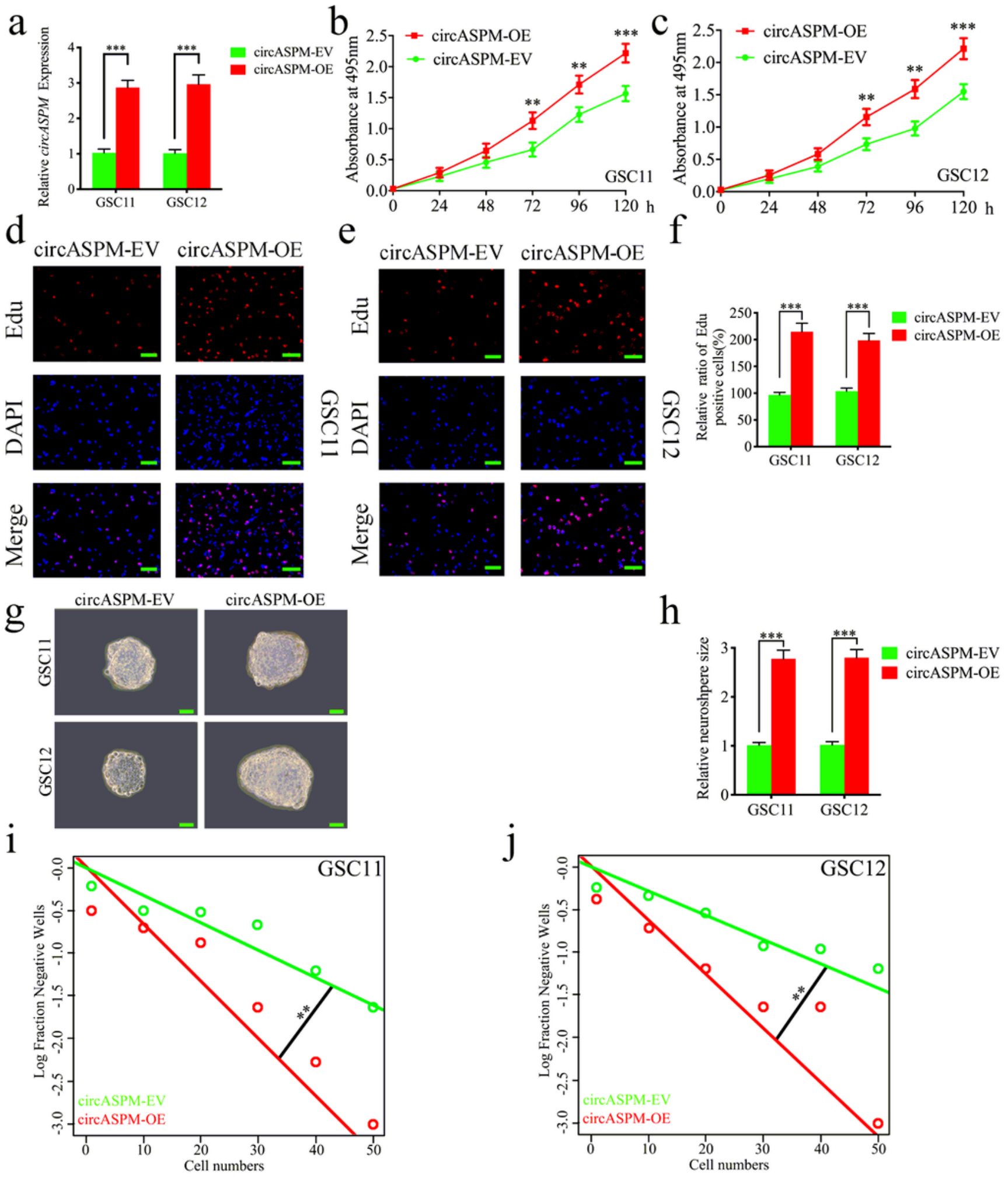

Figure 2

Overexpression of circASPM promoted the proliferation of GSCs in vitro. a, b: The expression of circASPM in GSC11 and GSC12 after transfection of the circASPM overexpression plasmids as measured by qPCR. c, f: circASPM overexpression significantly increased the proliferation of GSC11 and GSC12 in MTS 
assays. d, e, f: Proliferative capacity of tumor cells was inhibited after circASPM overexpression as measured by Edu assays. Scale bar $=50 \mu \mathrm{m} . \mathrm{g}, \mathrm{h}, \mathrm{i}, \mathrm{j}$ : Representative images of neurospheres and extreme limiting dilution assays showed tumor formation rate up-regulated after circASPM overexpression in GSC11 and GSC12. Scale bar $=50 \mu \mathrm{m}$. All data are shown as the mean $\pm \mathrm{SD}$ (three independent experiments). ${ }^{\star} \mathrm{P}<0.05 ; * \star \mathrm{P}<0.01 ; * \star * \mathrm{P}<0.001$

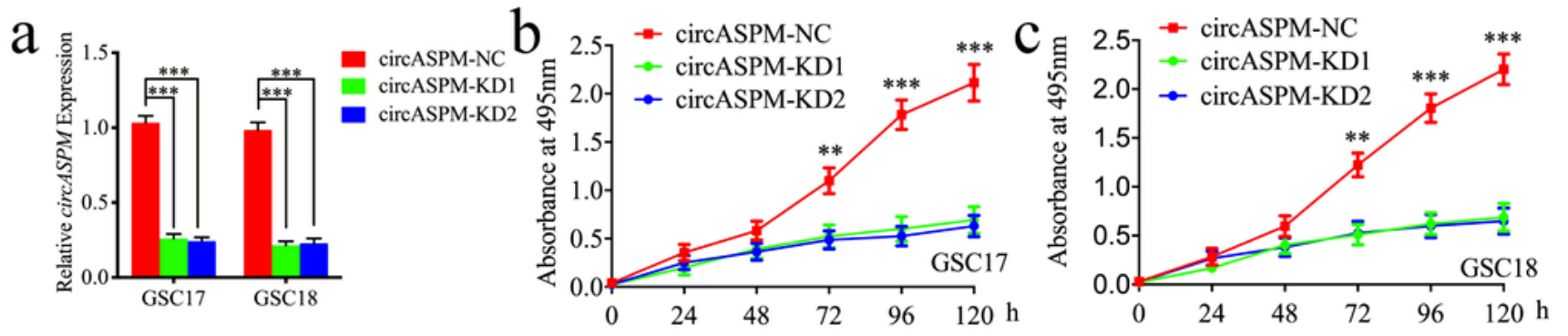

d circASPM-NC circASPM-KD1 circASPM-KD2
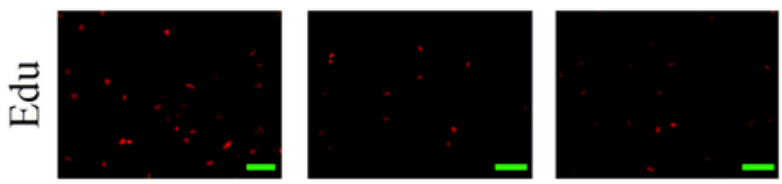

e
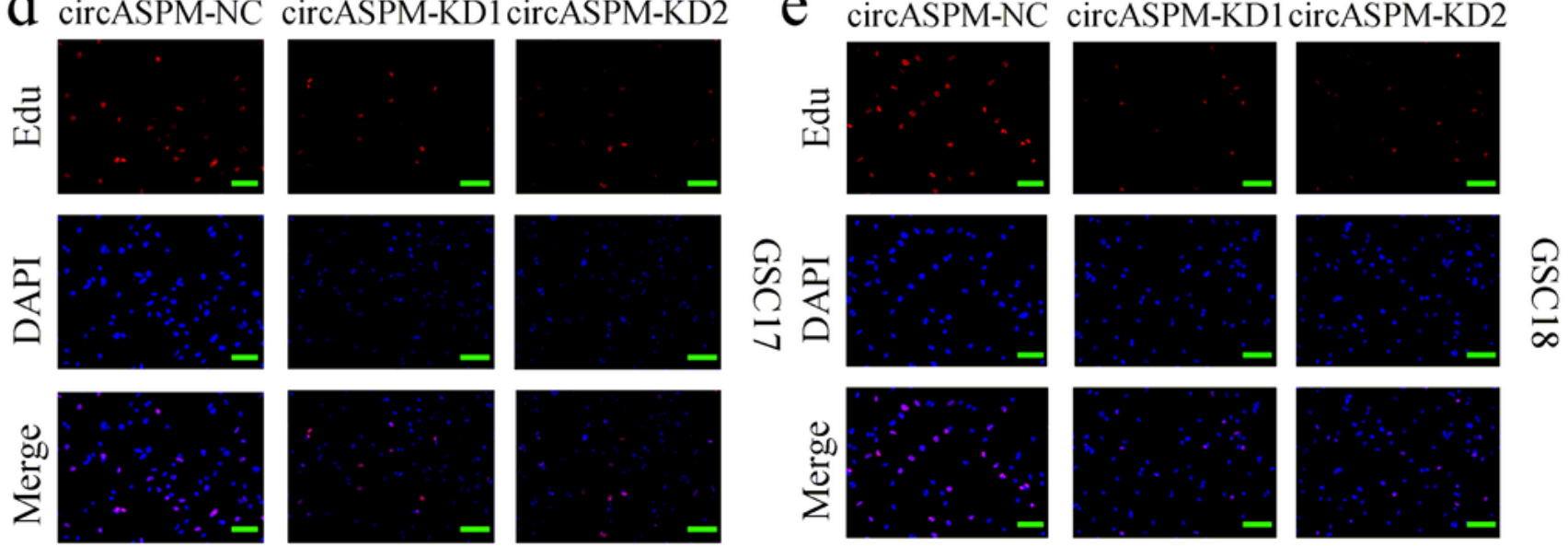

f

$\mathrm{g}$
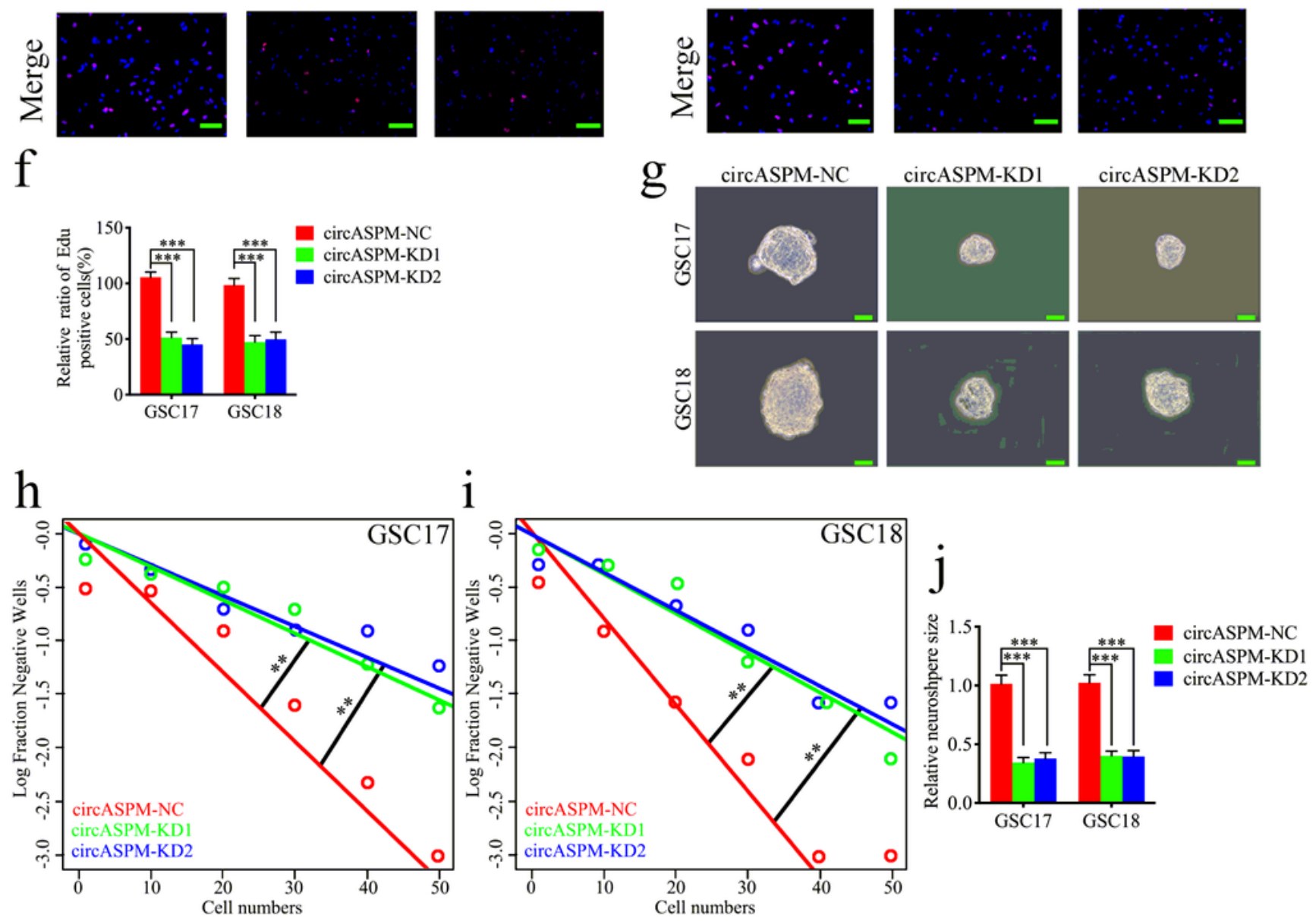

Figure 3 
Knockdown of circASPM suppressed the proliferation of GSCs in vitro. a: The expression of circASPM in GSC17 and GSC18 after transfection of circASPM-KD1, circASPM-KD2 or negative control as measured by qPCR. b, c: CircASPM knockdown significantly reduced the proliferation of GSC17 and GSC18 in MTS assays. d, e, f: CircASPM knockdown can significantly increase the proliferative capacity of GSC17 and GSC18 as measured by Edu assays. Scale bar $=50 \mu \mathrm{m} . \mathrm{g}, \mathrm{j}, \mathrm{h}$, i: Neurospheres formation assays and extreme limiting dilution assays showed that the tumor formation rates decreased after circASPM knockdown in GSC17 and GSC18. Scale bar $=50 \mu \mathrm{m}$. All data are shown as the mean \pm SD (three independent experiments). ${ }^{\star} \mathrm{P}<0.05 ;{ }^{* \star} \mathrm{P}<0.01 ; \star \star \star \mathrm{P}<0.001$. 
ha-miR-130b-3p 3r UACGGGAMAGUAU-AACGUGAC
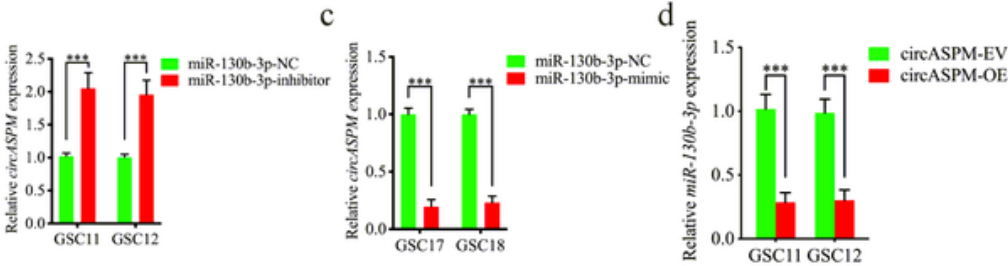

circASPM-mt $\quad 5$..ACAAAAGAACUGGU-AUGGUGAU.
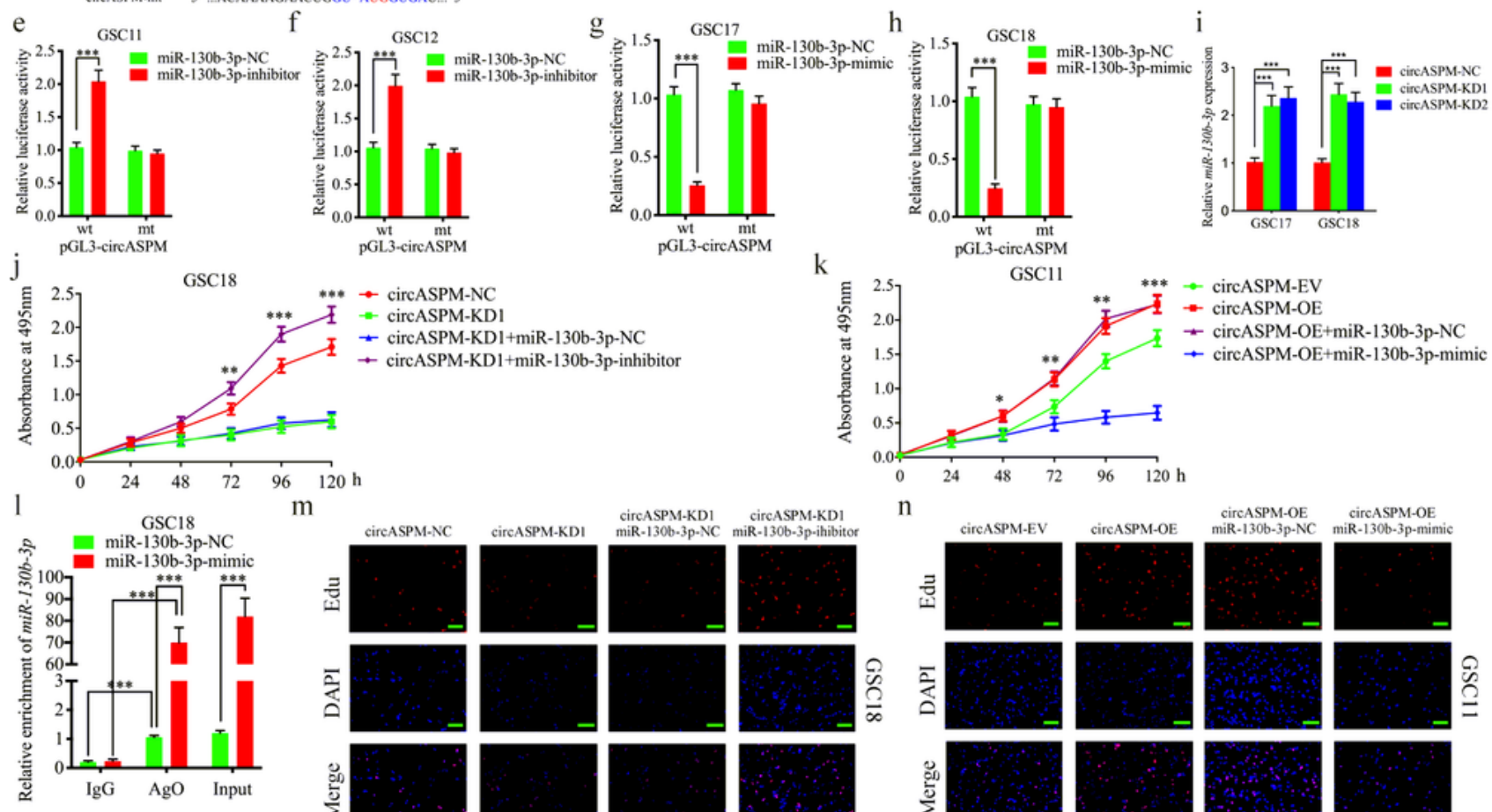

$\mathrm{m}$

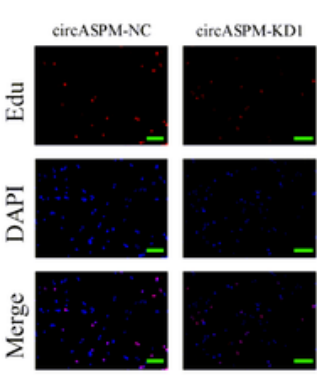

circASPM-KD1 circASPM-KDI
miR-1306-3p-NC
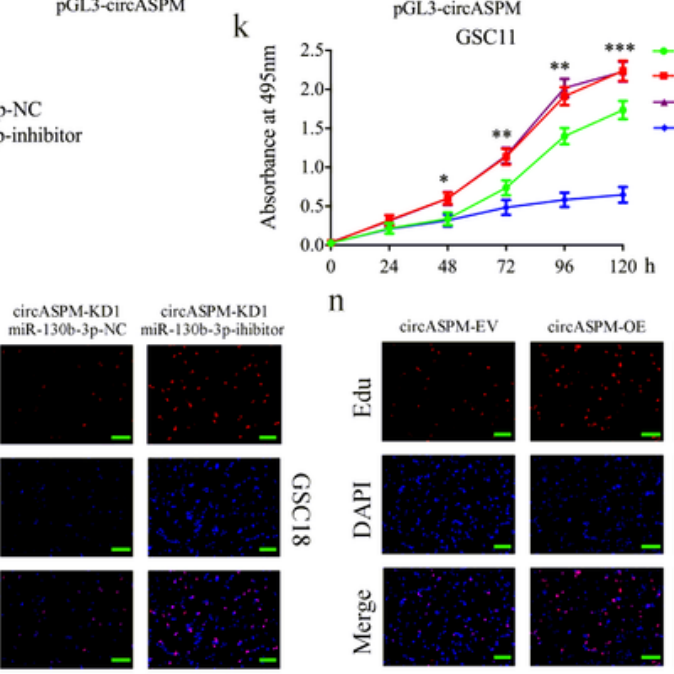

n

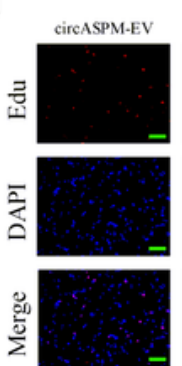

q
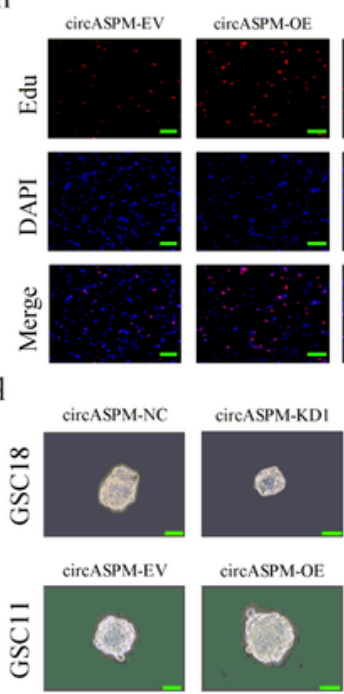

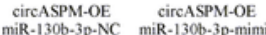
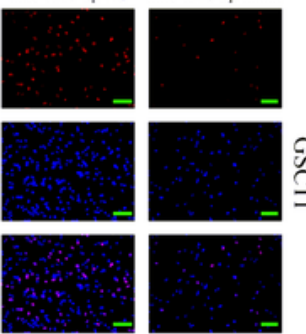

circASPM-KDI

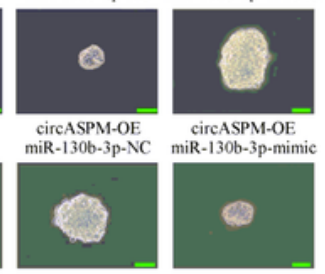

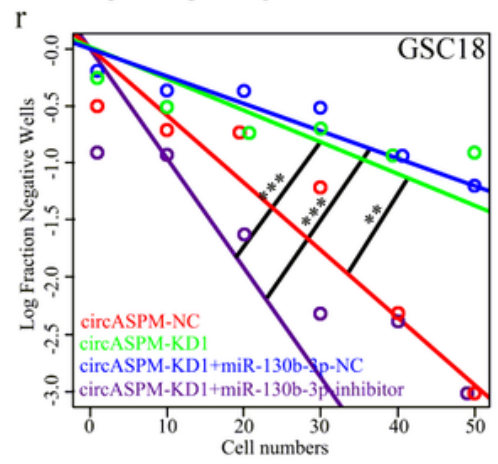
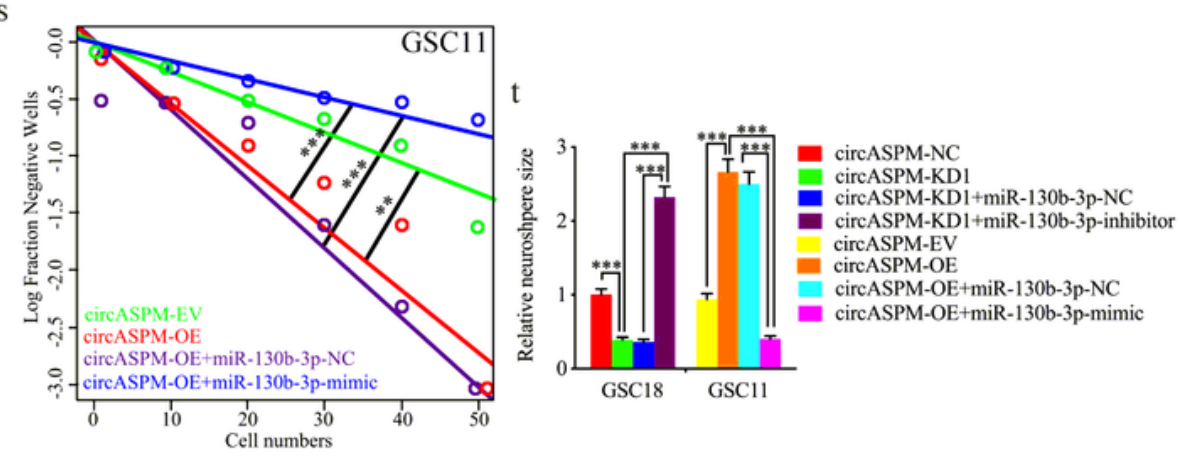

Figure 4

MiR-130b-3p could bind with circAPSM and mediated the function of GBM cells. a: The predicted binding site between circASPM and miR-130b-3p. b, c: MiR-130b-3p inhibitor treatment up-regulated the expression of circAPSM while mimic treatment down-regulated circASPM expression as measured by qPCR. $d$, i: The expression of miR-130b-3p was down-regulated after circAPSM overexpression while circASPM knockdown up-regulated miR-130b-3p expression as measured by qPCR. e, f, g, h: The 
luciferase reporter assays showed that miR-130b-3p mimic or inhibitor altered the luciferase promoter activities of circASPM. j, k: MTS assays showed that circASPM transfection of overexpression plasmids or si-circASPM affected GSCs viability and was reversed by miR-130b-3p mimic or inhibitor treatment, respectively. I, o: CircASPM and miR-130b-3p were effectively pulled down by anti-AGO2 antibodies compared to $\mathrm{IgG}$, and both enriched after miR-130b-3p mimic treatment in GSC18. $\mathrm{m}, \mathrm{n}, \mathrm{p}$ : The EDU assay showed that circASPM transfection of overexpression plasmids or si-circASPM affected GSCs proliferation capacity and was reversed by miR-130b-3p mimic or inhibitor treatment, respectively. Scale bar $=50 \mu \mathrm{m} . q, \mathrm{t}, \mathrm{r}, \mathrm{s}$ : In the neurosphere formation assays and extreme limiting dilution assays, circASPM transfection of overexpression plasmids or si-circASPM affected neurosphere growth capacity in GSCs and was reversed by miR-130b-3p mimic or inhibitor treatment, respectively. Scale bar $=50 \mu \mathrm{m}$. All data are shown as the mean $\pm S D$ (three independent experiments). ${ }^{*} P<0.05 ;{ }^{* \star} P<0.01 ;{ }^{* \star *} P<0.001$. 
a hsa-miR-130b-3p 3' UacGgGaAaguaguaAcGugac 5' $^{\prime}$ b

E2F1

5' ...UGGGGGGGCUCUAACUGCACUU... 3'

hsa-miR-130b-3p 3' UACGGGAAAGUAGUAACGUGAC $\quad 5^{\prime}$

wt-E2F1-3'-UTR 5' ...UGGGGGGGCUCUAACUGCACUU... 3'

hsa-miR-130b-3p $3^{\prime} \quad$ UACGGGAAAGUAGUAACGUGAC $\quad 5^{\prime}$

mt-E2F1-3'-UTR 5 ' ...UGGGGGGGCUCUAACACCAGAU... 3'

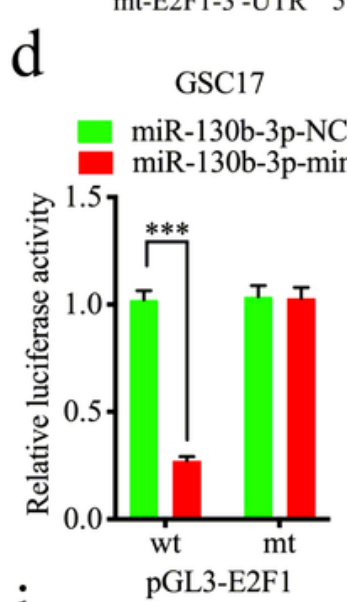

$\mathrm{e}$

GSC18

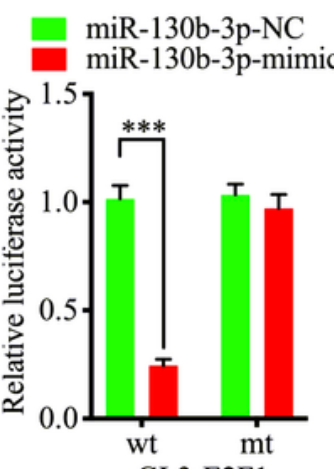

j

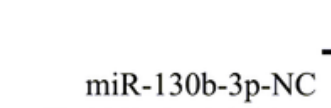

miR-130b-3p-inhibitor

E2F1

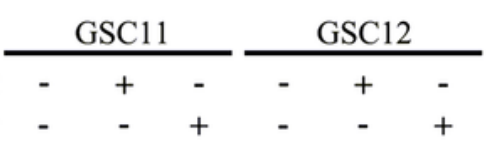

$\beta$-actin

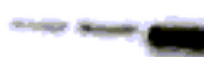

b

f
GSC11

miR-130b-3p-NC

miR-130b-3p-inhibitor

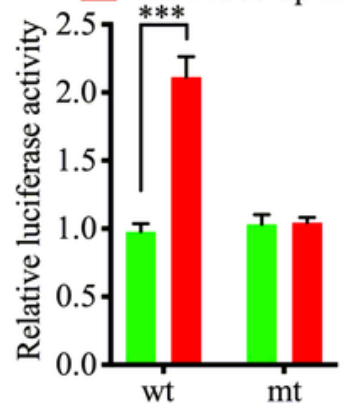

pGL3-E2F1
C
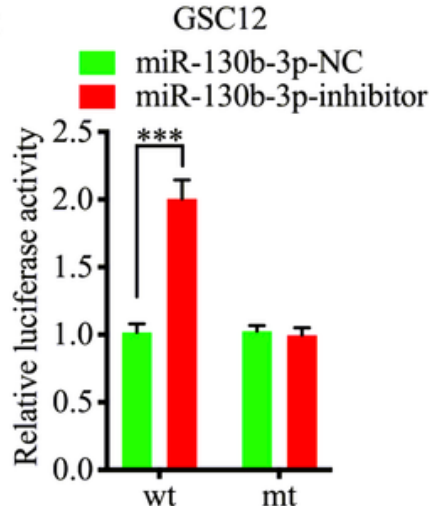

pGL3-E2F1
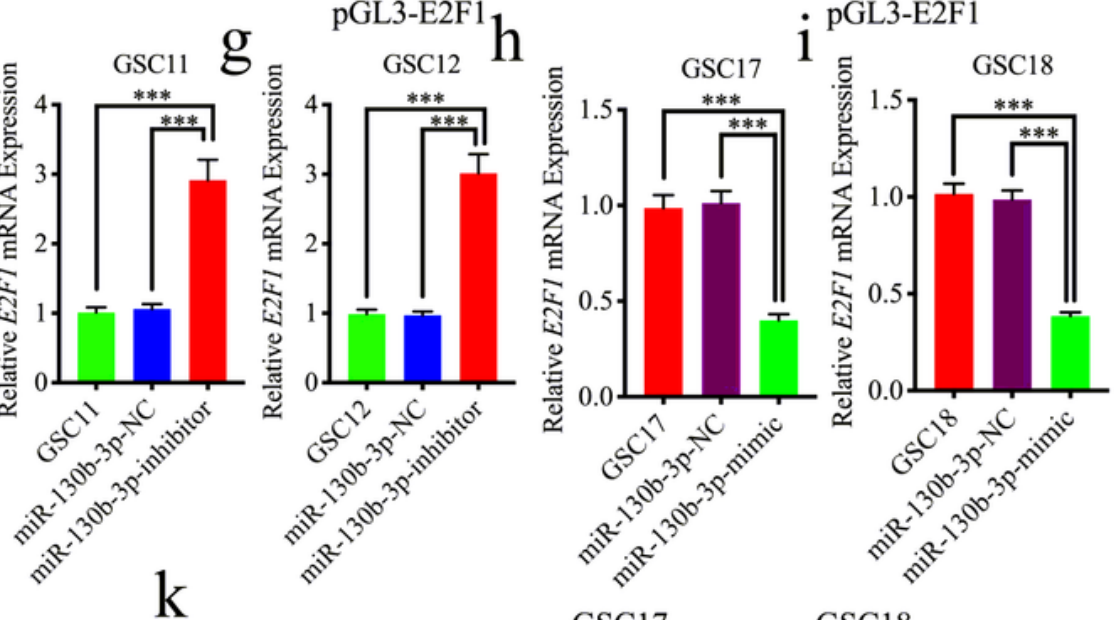

- $+-130 \mathrm{~b}-3 \mathrm{p}-\mathrm{NC}-+\quad-$ miR-130b-3p-mimic

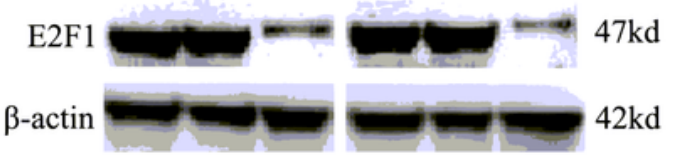

1
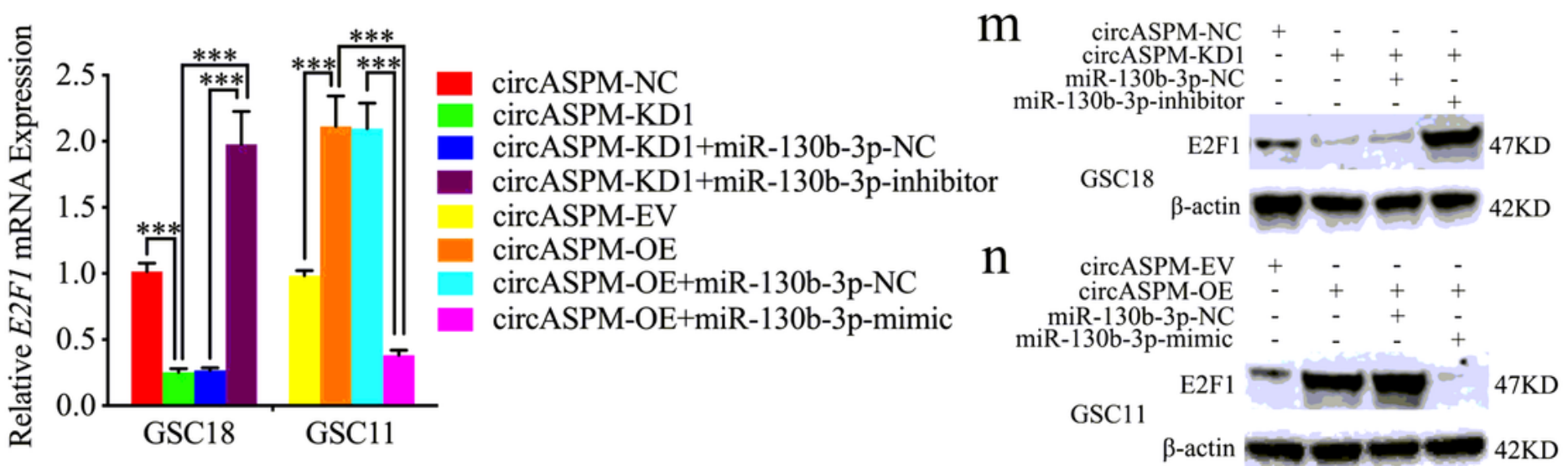

Figure 5

CircAPSM regulate E2F1 expression via miR-130b-3p sponging. a: Schematic diagram of the putative miR-130b-3p binding site in the 3'-UTR of E2F1. b, c, d, e: The luciferase reporter assays showed that miR130b-3p mimic or inhibitor altered the luciferase promoter activities of E2F1 f, g, h, i, j, k: qPCR and western blotting showed the expression of E2F1 in GSCs after miR-130b-3p mimic or inhibitor treatment. I, m, n: The decreased or increased expression of E2F1 in GSCs induced by cARF1 knockdown or 
overexpression was reversed by miR-130b-3p inhibitor or mimic treatment, as determined by western blotting and qPCR. All data are shown as the mean \pm SD (three independent experiments). ${ }^{*} \mathrm{P}<0.05$; ${ }^{\star \star} \mathrm{P}<$ $0.01 ; * \star * P<0.001$.

a

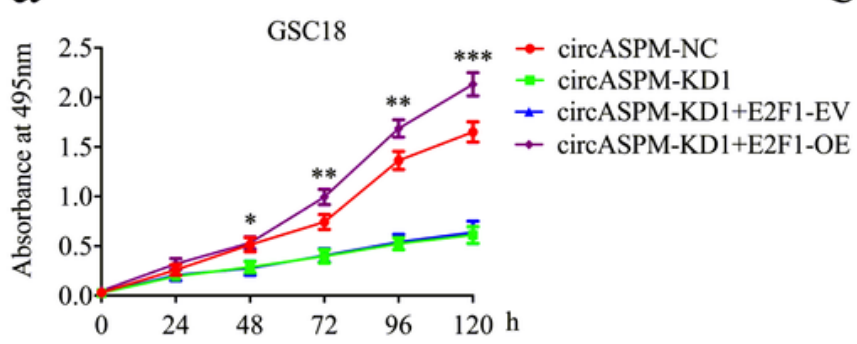

c
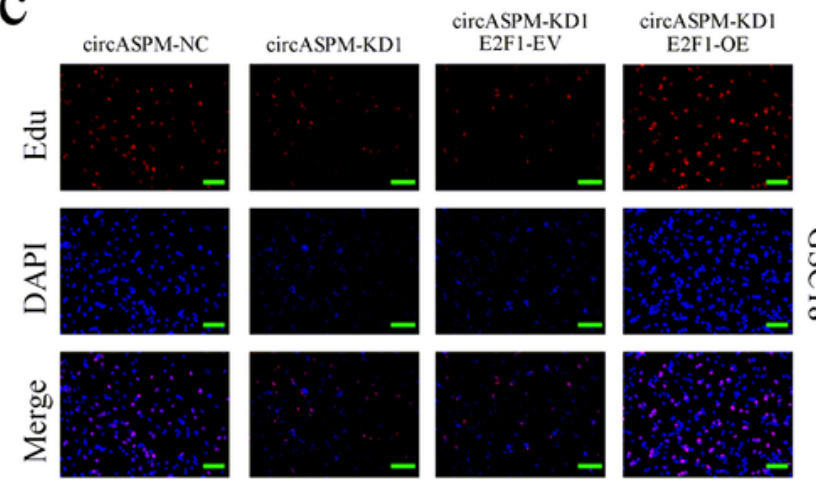

b

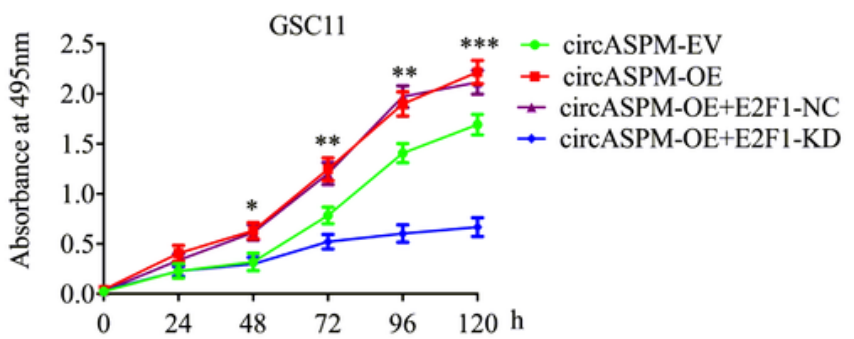

d

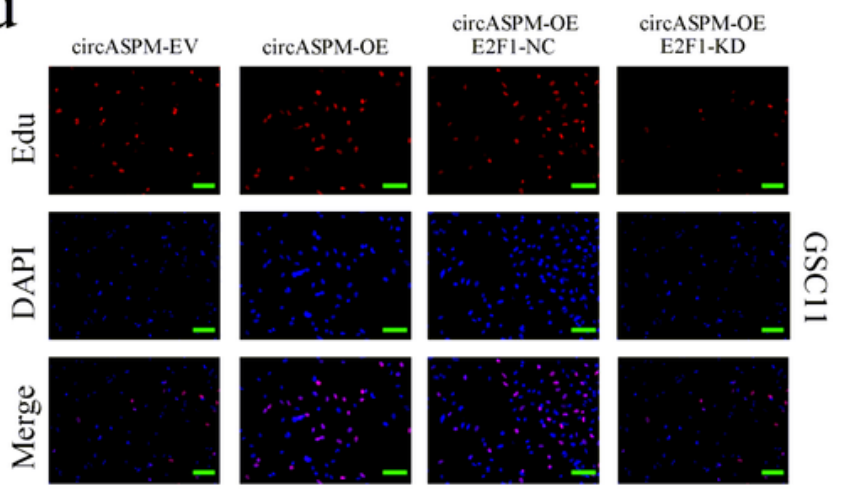

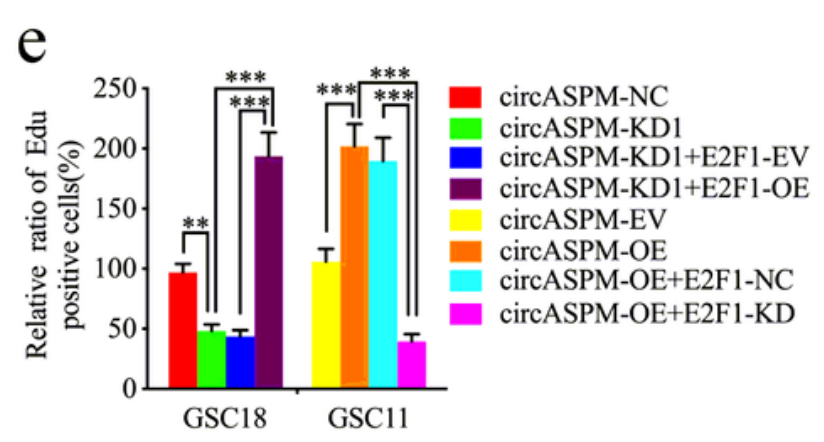
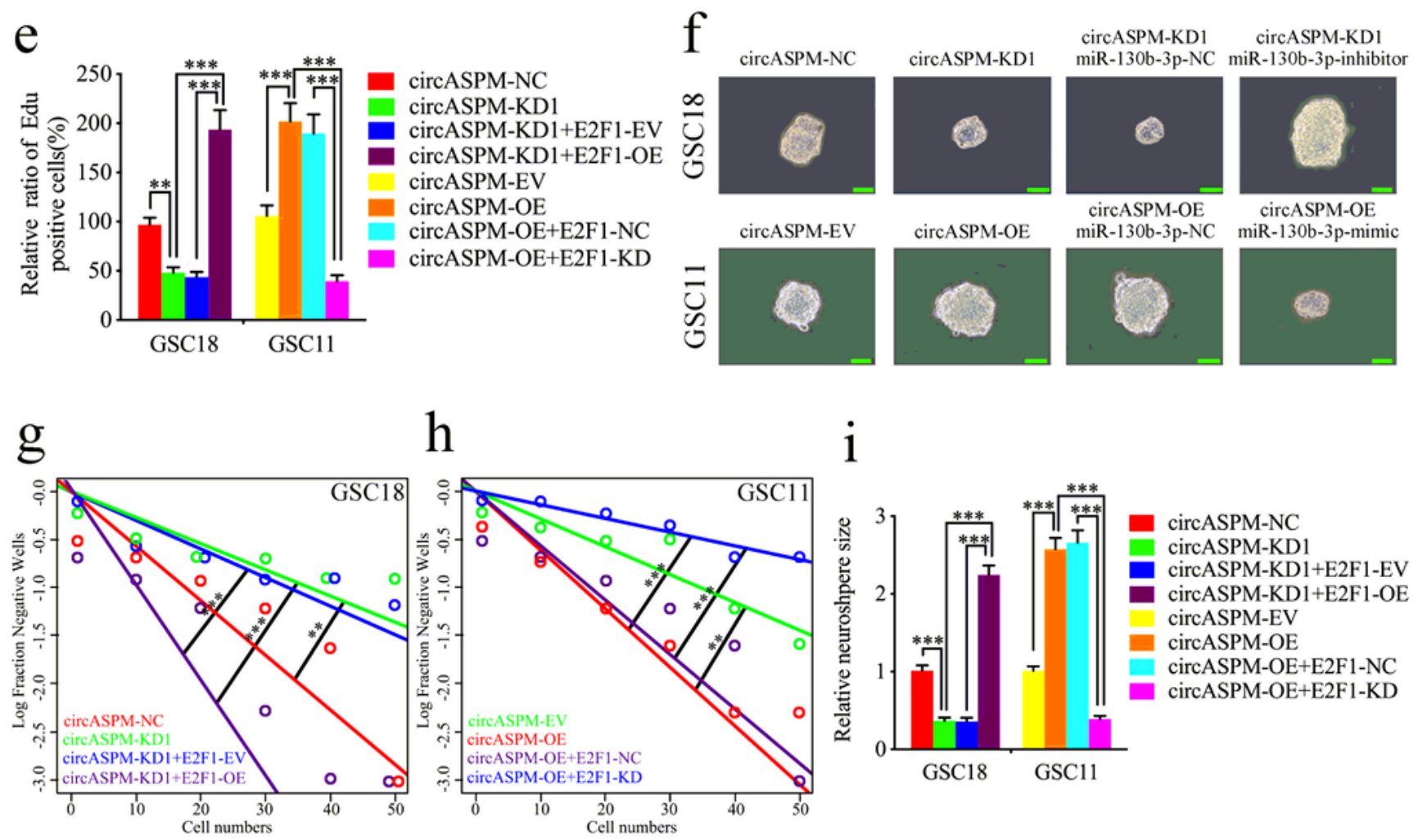

h

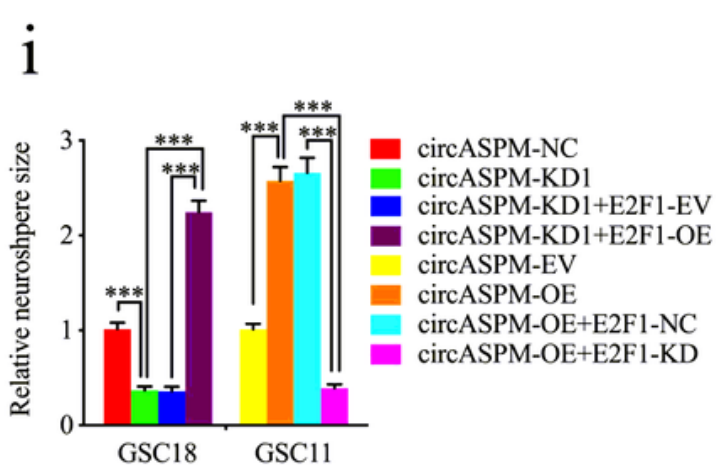

Figure 6

CircAPSM mediated GBM cells proliferation via E2F1expression. a,b: MTS assays showed that the GSCs viability regulated by circASPM knockdown or overexpression treatment were reversed by E2F1 
overexpression or knockdown, respectively. c, d, e: The EDU assay showed that the GSCs proliferation regulated by circASPM knockdown or overexpression treatment were reversed by E2F1 overexpression or knockdown, respectively. Scale bar $=50 \mu \mathrm{m} . \mathrm{f}, \mathrm{g}, \mathrm{h}, \mathrm{i}$ : The neurosphere formation assays and extreme limiting dilution assays showed that the circASPM transfection of overexpression plasmids or sicircASPM affected neurosphere growth capacity in GSCs and was reversed by miR-130b-3p E2F1 mimic or inhibitor treatment, respectively. Scale bar $=50 \mu \mathrm{m}$. All data are shown as the mean $\pm \mathrm{SD}$ (three independent experiments). ${ }^{*} \mathrm{P}<0.05 ;{ }^{*} \mathrm{P}<0.01 ; * \star * \mathrm{P}<0.001$.

a

GSC18

circASPM-NC

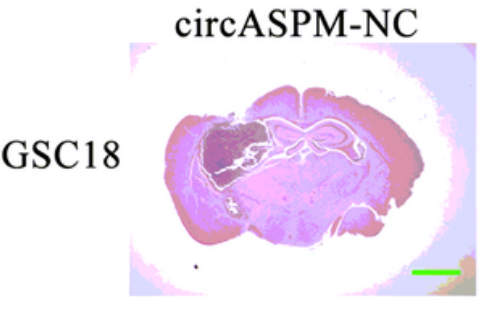

d

g

HE

E2F1

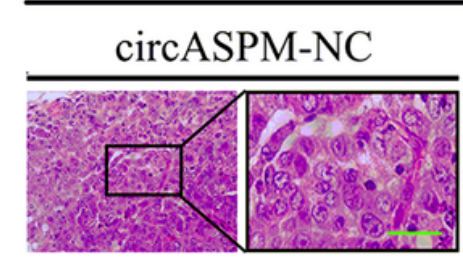

GSC18

GSC11

circASPM-EV
circASPM-KD1

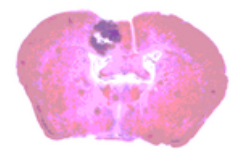

e
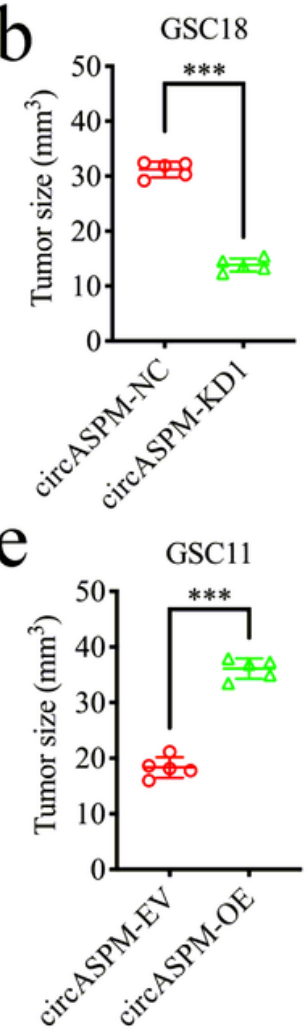

circASPM-OE

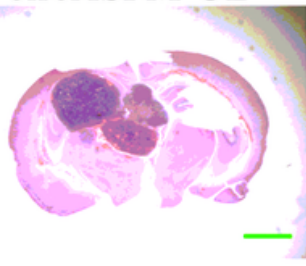

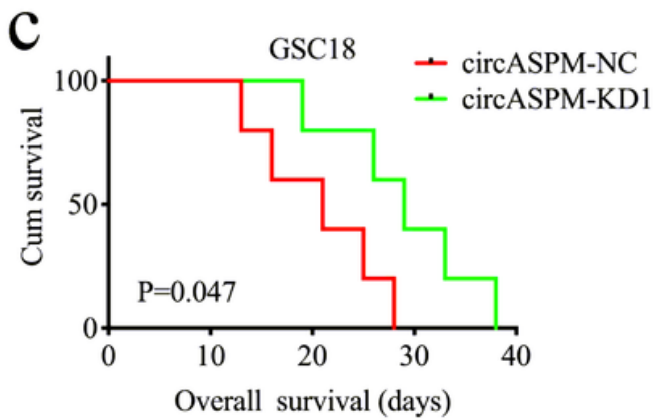

f

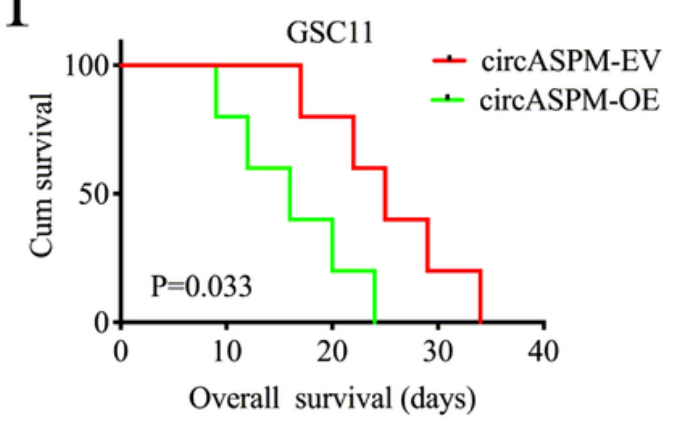

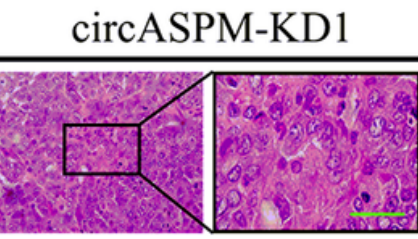

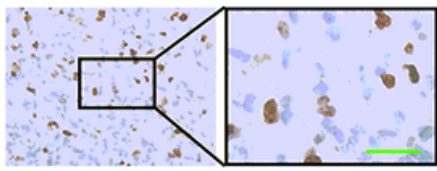

Ki-67

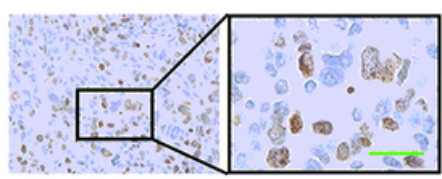

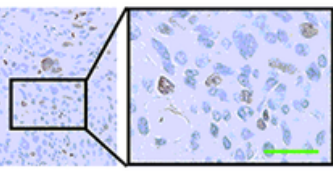

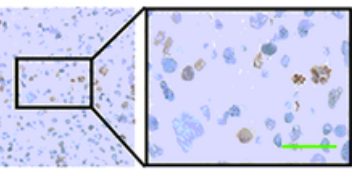

GSC11

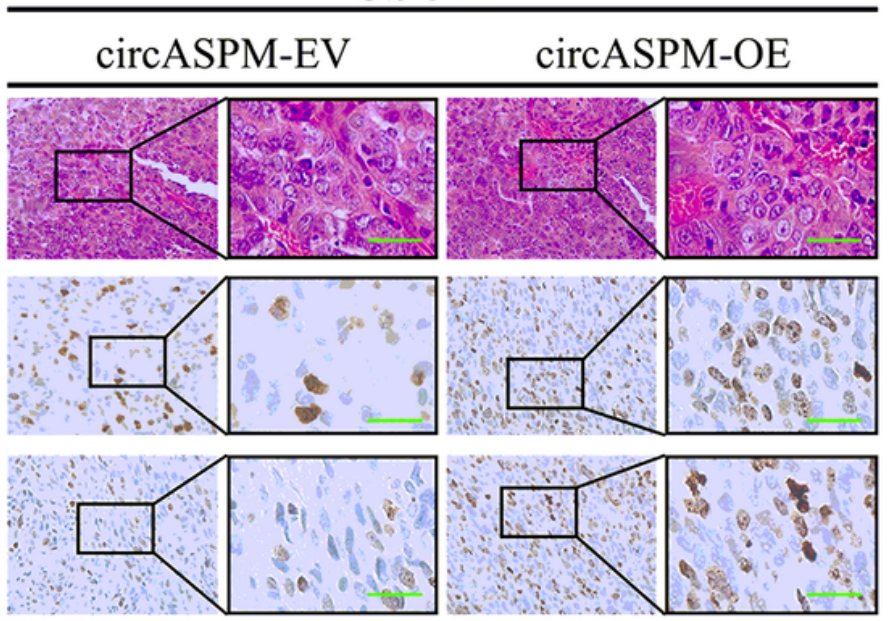

Figure 7

CircAPSM mediated GBM cells proliferation via E2F1expression. a, b: H\&E-stained brain sections of mice with circAPSM knockdown in GSC18 were transplanted intracranially. The brain was harvested on the 
15th day after transplantation. CircAPSM knockdown significantly inhibits tumor growth in vivo. Scale bar $=1 \mathrm{~mm}$. c: Kaplan-Meier survival curve of circAPSM knockdown in GSC18 mice. d, e: H\&E-stained brain sections of mice with circAPSM overexpression in GSC11 were transplanted intracranially. The brain was harvested on the 15th day after transplantation. CircAPSM overexpression significantly enhances tumor growth in vivo. Scale bar $=1 \mathrm{~mm}$. f: Kaplan-Meier survival curve of circAPSM overexpression in GSC11. g, m: Representative immunohistochemical staining showed the changes in $\mathrm{H} \& \mathrm{E}, \mathrm{E} 2 \mathrm{~F} 1$, and ki-67 staining in circAPSM overexpression and knockdown orthotopic xenograft models. Scale bar $=50 \mu \mathrm{m}$. All data are shown as the mean $\pm \mathrm{SD}$ (three independent experiments). ${ }^{*} \mathrm{P}<0.05 ; * \star \mathrm{P}<$ $0.01 ; * \star * P<0.001$.

\section{Supplementary Files}

This is a list of supplementary files associated with this preprint. Click to download.

- supplementaryfigure1.tif

- SupplementaryTable1.docx

- SupplementaryTable2.docx 